\title{
Existence and uniqueness criteria for the higher-order Hilfer fractional boundary value problems at resonance
}

\section{Yousef Gholami ${ }^{1 *}$ (D)}

\section{"Correspondence:}

y_gholami@sut.ac.ir

1 Department of Applied

Mathematics, Sahand University of

Technology, Sahand New Town,

51335-1996, Tabriz, Iran

\section{Springer}

\begin{abstract}
This investigation is devoted to the study of a certain class of coupled systems of higher-order Hilfer fractional boundary value problems at resonance. Combining the coincidence degree theory with the Lipschitz-type continuity conditions on nonlinearities, we present some existence and uniqueness criteria. Finally, to practically implement the obtained theoretical criteria, we give an illustrative application.
\end{abstract}

MSC: Primary 26A33; 34A34; 35B34; secondary 34A08; 34A12; 47H11

Keywords: Hilfer fractional boundary value problems; Resonance; Coupled systems; Coincidence degree theory; Existence; Uniqueness

\section{Introduction}

In this paper, we study the coupled systems of the higher-order Hilfer fractional differential equations

$$
\begin{cases}D_{a^{+}}^{\alpha, \beta} u(t)=\Phi\left(t, v, D_{a^{+}}^{1-(n-\alpha)(1-\beta), \beta} v, \ldots, D_{a^{+}}^{n-(n-\alpha)(1-\beta)-1, \beta} v\right), & a<t<b, \\ D_{a^{+}}^{\alpha, \beta} v(t)=\Psi\left(t, u, D_{a^{+}}^{1-(n-\alpha)(1-\beta), \beta} u, \ldots, D_{a^{+}}^{n-(n-\alpha)(1-\beta)-1, \beta} u\right), & a<t<b,\end{cases}
$$

subject to the boundary conditions

$$
\begin{cases}D_{a^{+}}^{n-(n-\alpha)(1-\beta)-i, \beta} u(a)=0, & D_{a^{+}}^{n-(n-\alpha)(1-\beta)-1, \beta} u(a)=D_{a^{+}}^{n-(n-\alpha)(1-\beta)-1, \beta} u(b), \\ D_{a^{+}}^{n-(n-\alpha)(1-\beta)-i, \beta} v(a)=0, & D_{a^{+}}^{n-(n-\alpha)(1-\beta)-1, \beta} v(a)=D_{a^{+}}^{n-(n-\alpha)(1-\beta)-1, \beta} v(b),\end{cases}
$$

for $i=2,3, \ldots, n$.

The general assumptions of the coupled system (1.1)-(1.2) are as follows:

$\left(A_{1}\right) D_{a^{+}}^{\alpha, \beta}$ stands for the Hilfer fractional derivative of order $n-1<\alpha \leq n, n \in \mathbb{N}_{2}$, and type $0 \leq \beta \leq 1$.

$\left(A_{2}\right) \Phi, \Psi:[a, b] \times \mathbb{R}^{n} \rightarrow \mathbb{R}$ are continuous functions governing the nonlinearities.

$\left(A_{3}\right) I_{a^{+}}^{j} g(t)=0, j=1,2, \ldots, n$, and $I_{a^{+}}^{1-\beta(n-\alpha)} g(t)=0, g=u, v$, where $I_{a^{+}}^{\mu}$ stands for the Riemann-Liouville fractional integral of order $\mu$.

(c) The Author(s) 2020. This article is licensed under a Creative Commons Attribution 4.0 International License, which permits use, sharing, adaptation, distribution and reproduction in any medium or format, as long as you give appropriate credit to the original author(s) and the source, provide a link to the Creative Commons licence, and indicate if changes were made. The images or other third party material in this article are included in the article's Creative Commons licence, unless indicated otherwise in a credit line to the material. If material is not included in the article's Creative Commons licence and your intended use is not permitted by statutory regulation or exceeds the permitted use, you will need to obtain permission directly from the copyright holder. To view a copy of this licence, visit http://creativecommons.org/licenses/by/4.0/. 
Since the second half of the seventeenth century by now, almost over 370 years, applicability potential of the differential/difference equations and their mutual inverses, integral/finite sum equations in description of the natural phenomena besides theoretical sciences tied up with mathematics have been proved. Meanwhile, the fractional differential calculus that includes arbitrary-order differential/difference and integral/finite sum operators, as a result of more rigorous frame to describe biological, chemical, and physical models of natural phenomena, possesses an elegant position in comparison with the integer-order differential calculus. Reasonability of our claim can be verified in the monographs $[4,6,7,18,26,27,33-38]$.

Restricting ourselves to the investigation of the solvability of fractional-order differential equations opens an independent wide research world in front of us. As some of the generally known techniques to solve a given fractional differential equation, we may mention the fixed point theory (via Green functions, controlling the growth of nonlinearities or measures of noncompactness), the operational calculus, the approximation theory, and the coincidence degree theory. Here we suggest (absolutely not comprehensive) a collection of the pioneering research papers $[1-3,5,8-14,17,21,23-25,32,40,42-47]$, and $[19,22,28-31]$, respectively, and cited bibliography for more consultation of the interested followers.

Among this variety, we are interested in the investigation of solvability of higher-order generalized fractional differential systems using coincidence degree theory due to Mawhin [33]. More precisely, we are going to study fractional coupled systems at resonance. To specify the concept of resonance, let us consider the differential system

$$
\mathfrak{L} u=\mathfrak{F}, \quad \mathfrak{B} \mathfrak{C} u=0,
$$

in which $\mathfrak{L}, \mathfrak{F}$, and $\mathfrak{B C}$ denote a differential operator, nonlinearity, and boundary conditions of the system, respectively. We say that the differential system (1.3) is of resonance category if in the corresponding homogenous differential system

$$
\mathfrak{L} u=0, \quad \mathfrak{B C} u=0,
$$

the differential operator $\mathfrak{L}$ is invertible with respect to the boundary conditions $\mathfrak{B C}$, that is, there is at least one nontrivial solution for the homogenous differential system (1.4). Otherwise, we are concerned with the nonresonance case. For some of the most motivating research works on the fractional-order resonance phenomenon, we refer to [22, 28-31].

The authors in [16] considered the coupled resonant system of the higher-order Caputotype fractional $\Delta$-difference boundary value problems

$$
\begin{aligned}
& \left\{\begin{array}{l}
\Delta_{*}^{\alpha} y(t)=f\left(t+N-\alpha-2, z, \Delta z, \Delta^{2} z, \ldots, \Delta^{N-1} z\right), \\
\quad t=a, a+1, \ldots, b, \\
\Delta_{*}^{\alpha} z(t)=g\left(t+N-\alpha-2, y, \Delta y, \Delta^{2} y, \ldots, \Delta^{N-1} y\right),
\end{array}\right. \\
& \begin{cases}\Delta^{N-i} y(a+N-\alpha-2)=0, & \Delta^{N-1} y(a+N-\alpha-2)=\Delta^{N-1} y(b+N-\alpha-1), \\
\Delta^{N-i} z(a+N-\alpha-2)=0, & \Delta^{N-1} z(a+N-\alpha-2)=\Delta^{N-1} z(b+N-\alpha-1),\end{cases}
\end{aligned}
$$


where $i=2,3, \ldots, N, N-1<\alpha \leq N, N \in \mathbb{N}_{2}$, and $a \in \mathbb{Z}_{1}, b \in \mathbb{Z}_{2}$ with $a<b, \Delta_{*}^{\alpha}$ denotes the Caputo-type fractional $\Delta$-difference of order $\alpha>0$, and $f, g: \mathbb{N}_{a+N-\alpha-2}^{b+N-\alpha-1} \times \mathbb{R}^{N} \rightarrow \mathbb{R}$ are continuous functions. Using the coincidence degree theory, the authors obtained some existence and uniqueness criteria for the discrete fractional coupled system (1.5)-(1.6).

The authors in [19] studied the following higher-order Caputo fractional resonant system:

$$
\begin{cases}D_{0^{+}}^{\alpha} u(t)=f\left(t, v, v^{\prime}, v^{\prime \prime}, \ldots, v^{(n-1)}\right), & 0<t<1 \\ D_{0^{+}}^{\alpha} v(t)=g\left(t, u, u^{\prime}, u^{\prime \prime}, \ldots, u^{(n-1)}\right), & 0<t<1\end{cases}
$$

subject to the boundary conditions

$$
\left\{\begin{array}{l}
u(a)=u^{\prime}(0)=u^{\prime \prime}(0)=\cdots=u^{(n-2)}(0)=0, \quad u^{(n-1)}(0)=u^{(n-1)}(1), \\
v(a)=v^{\prime}(0)=v^{\prime \prime}(0)=\cdots=v^{(n-2)}(0)=0, \quad v^{(n-1)}(0)=v^{(n-1)}(1) .
\end{array}\right.
$$

Thanks to the coincidence degree theory, they obtained existence criteria for at least one solution of this fractional-order coupled system.

Also, the authors in [48] concentrated on the higher-order Riemann-Liouville fractional resonant boundary value problem

$$
\left\{\begin{array}{l}
D_{0^{+}}^{\alpha} u(t)=f\left(t, u(t), D_{0^{+}}^{\alpha-n+1} u(t), D_{0^{+}}^{\alpha-n+2} u(t), \ldots, D_{0^{+}}^{\alpha-1} u(t)\right)+e(t), \quad 0<t<1, \\
I_{0^{+}}^{n-\alpha} u(0)=D_{0^{+}}^{\alpha-n+1} u(0)=\cdots=D_{0^{+}}^{\alpha-2} u(0)=0, \quad u(1)=\sigma u(\eta),
\end{array}\right.
$$

where $n-1<\alpha \leq n, n \in \mathbb{N}_{2}, \sigma \in(0,+\infty)$, and $\eta \in(0,1)$ with $\sigma \eta^{\alpha-1}=1, e \in L[0,1]$, and $f:[0,1] \times \mathbb{R}^{n} \rightarrow \mathbb{R}$ is a continuous function. Similarly to the discrete fractional coupled system (1.5)-(1.6) and the Caputo fractional resonant system (1.7)-(1.8), some existence and uniqueness criteria were obtained via coincidence degree theory. The aforementioned research papers are the main motivation that led us to study the Hilfer fractional coupled system (1.1)-(1.2).

The paper is organized as follows. Some standard definitions and technical lemmas related to the fractional calculus operators, a quick overview on the coincidence degree theory, and demonstration why the Hilfer fractional differential system (1.1)-(1.2) is called a resonant differential system are given in the Sect. 2 . Section 3, the main body of the investigation, includes some existence and uniqueness criteria for the Hilfer fractional resonant system (1.1)-(1.2). In this section, combining the coincidence degree theory with some controls on the nonlinearities $\Phi$ and $\Psi$, we prove the existence of at least one solution of the fractional coupled system (1.1)-(1.2), and imposing another conditions on these nonlinearities, we present a uniqueness criterion. In Sect. 4, we present an illustrative example to justify the obtained theoretical results. The last section of this paper deals with the novelty and some advantages of studying the Hilfer fractional derivatives in differential equations. Furthermore, we suggest in this section some research lines for the future work on the topic. 


\section{Technical requirements}

This section begins with a quick overview on those parts of the fractional calculus that will be needed in this paper. So, we start with the definitions of the Riemann-Liouville fractional integrals and derivatives.

Definition 2.1 ([26]) The left- and right-sided Riemann-Liouville fractional integrals of order $\alpha \geq 0$ for a function $f \in L^{1}[a, b]$ are given by

$$
\mathcal{I}_{a^{+}\left(b_{-}-\right.}^{\alpha} f(t)= \begin{cases}\mathcal{I}_{a^{+}}^{\alpha} f(t)=\frac{1}{\Gamma(\alpha)} \int_{a}^{t}(t-s)^{\alpha-1} f(s) d s, & \alpha>0, \\ \mathcal{I}_{b-}^{\alpha} f(t)=\frac{1}{\Gamma(\alpha)} \int_{t}^{b}(s-t)^{\alpha-1} f(s) d s, & \alpha>0, \\ f(t), & \alpha=0 .\end{cases}
$$

Definition 2.2 ([26]) The left- and right-sided Riemann-Liouville fractional derivatives of order $\alpha \geq 0$ for a function $f \in A C^{n}(a, b)$ are defined by

$$
\begin{aligned}
& \mathcal{D}_{a^{+}\left(b_{-}\right)}^{\alpha} f(t) \\
& \quad= \begin{cases}\mathcal{D}_{a^{+}}^{\alpha} f(t):=\left(\frac{d^{n}}{d t^{n}}\right) \mathcal{I}_{a^{+}}^{n-\alpha} f(t)=\frac{1}{\Gamma(n-\alpha)}\left(\frac{d^{n}}{d t^{n}}\right) \int_{a}^{t}(t-s)^{n-\alpha-1} f(s) d s, & \alpha>0, \\
\mathcal{D}_{b-}^{\alpha} f(t):=\left(\frac{d^{n}}{d t^{n}}\right) \mathcal{I}_{b_{-}}^{n-\alpha} f(t)=\frac{(-1)^{n}}{\Gamma(n-\alpha)}\left(\frac{d^{n}}{d t^{n}}\right) \int_{t}^{b}(s-t)^{n-\alpha-1} f(s) d s, & \alpha>0, \\
f(t), & \alpha=0,\end{cases}
\end{aligned}
$$

where $n=[\alpha]+1$.

Interchanging the affection position of the $n$ th-order derivative $\frac{d^{n}}{d t^{n}}$ as follows gives us the left- and right-sided Caputo fractional derivatives

$$
\begin{aligned}
& { }^{c} \mathcal{D}_{a^{+}\left(b_{-} f\right.}^{\alpha} f(t) \\
& = \begin{cases}{ }^{c} \mathcal{D}_{a^{+}}^{\alpha} f(t):=\mathcal{I}_{a^{+}}^{n-\alpha}\left(\frac{d^{n}}{d t^{n}} f\right)(t)=\frac{1}{\Gamma(n-\alpha)} \int_{a}^{t}(t-s)^{n-\alpha-1}\left(\frac{d^{n}}{d t^{n}} f\right)(s) d s, & \alpha>0, \\
{ }^{c} \mathcal{D}_{b-}^{\alpha} f(t):=\mathcal{I}_{b_{-}}^{n-\alpha}\left(\frac{d^{n}}{d t^{n}} f\right)(t)=\frac{(-1)^{n}}{\Gamma(n-\alpha)} \int_{t}^{b}(s-t)^{n-\alpha-1}\left(\frac{d^{n}}{d t^{n}} f\right)(s) d s, & \alpha>0, \\
f(t), & \alpha=0 .\end{cases}
\end{aligned}
$$

The power rules of the Riemann-Liouville fractional operators are given in the following lemma.

Lemma 2.3 ([26]) Let $n-1<\alpha \leq n, n \in \mathbb{N}$. Then for each $\beta>-1$, we have

$$
\begin{aligned}
& \left(\mathcal{I}_{a^{+}}^{\alpha}(t-a)^{\beta}\right)(x)=\frac{\Gamma(\beta+1)}{\Gamma(\beta+\alpha+1)}(x-a)^{\beta+\alpha}, \\
& \left(\mathcal{I}_{b_{-}}^{\alpha}(b-t)^{\beta}\right)(x)=\frac{\Gamma(\beta+1)}{\Gamma(\beta+\alpha+1)}(b-x)^{\beta+\alpha}, \\
& \left(\mathcal{D}_{a^{+}}^{\alpha}(t-a)^{\beta}\right)(x)=\frac{\Gamma(\beta+1)}{\Gamma(\beta-\alpha+1)}(x-a)^{\beta-\alpha}, \\
& \left(\mathcal{D}_{b_{-}}^{\alpha}(b-t)^{\beta}\right)(x)=\frac{(-1)^{n} \Gamma(\beta+1)}{\Gamma(\beta-\alpha+1)}(b-x)^{\beta-\alpha} .
\end{aligned}
$$

Now we are ready to define the Hilfer fractional derivatives and their basic properties. 
Definition 2.4 ([18, 41]) Let $n-1<\alpha \leq n, n \in \mathbb{N}$, and $0 \leq \beta \leq 1$. Then the left- and rightsided Hilfer fractional derivatives of order $\alpha$ and type $\beta$ are defined as follows:

$$
\mathcal{D}_{a^{+}\left(b_{-}\right)}^{\alpha, \beta} f(t)= \begin{cases}\mathcal{D}_{a^{+}}^{\alpha, \beta} f(t):=\left(\mathcal{I}_{a^{+}}^{\beta(n-\alpha)} \mathcal{D}_{a^{+}}^{\alpha+\beta(n-\alpha)} f\right)(t), & t>a, \\ \mathcal{D}_{b_{-}}^{\alpha, \beta} f(t):=\left(\mathcal{I}_{b_{-}}^{\beta(n-\alpha)} \mathcal{D}_{b_{-}}^{\alpha+\beta(n-\alpha)} f\right)(t), & t<b .\end{cases}
$$

Note for $\beta=0$, the left- and right-sided Hilfer fractional derivatives (2.8) reduce to the left- and right-sided Riemann-Liouville fractional derivatives (2.2), whereas for $\beta=1$, they reduce to the left- and right-sided Caputo fractional derivatives (2.3).

Next, we present the inversion rules of the Hilfer fractional derivatives.

Lemma 2.5 ([41]) Let $n-1<\alpha \leq n, n \in \mathbb{N}, 0 \leq \beta \leq 1$, and let the assumption $\left(A_{3}\right)$ hold. If $f \in L(a, b)$ and $\mathcal{I}_{a^{+}}^{(n-\alpha)(1-\beta)} f \in A C^{n-1}[a, b]$, then

$$
\begin{aligned}
& \left(\mathcal{D}_{a^{+}}^{\alpha, \beta} \mathcal{I}_{a^{+}}^{\alpha} f\right)(t)=f(t), \\
& \left(\mathcal{I}_{a^{+}}^{\alpha} \mathcal{D}_{a^{+}}^{\alpha, \beta} f\right)(t)=f(t)-\sum_{k=0}^{n-1} \frac{(t-a)^{k-(n-\alpha)(1-\beta)}}{\Gamma(k-(n-\alpha)(1-\beta)+1)} \cdot c_{k},
\end{aligned}
$$

where

$$
c_{k}:=\lim _{t \rightarrow a^{+}} \frac{d^{k}}{d x^{k}}\left(\mathcal{I}_{a^{+}}^{(n-\alpha)(1-\beta)} f\right)(t)
$$

Based on the power rules (2.4)-(2.7) in Lemma 2.3, we give the following power rules for the Hilfer fractional derivatives without proof.

Lemma 2.6 Let $n-1<\alpha \leq n, n \in \mathbb{N}$, and $0 \leq \beta \leq 1$. Then, for each $\gamma>-1$,

$$
\begin{aligned}
& \left(\mathcal{D}_{a^{+}}^{\alpha, \beta}(t-a)^{\gamma}\right)(x)=\frac{\Gamma(\gamma+1)}{\Gamma(\gamma-\alpha+1)}(x-a)^{\gamma-\alpha}, \\
& \left(\mathcal{D}_{b_{-}}^{\alpha, \beta}(b-t)^{\gamma}\right)(x)=\frac{(-1)^{n} \Gamma(\gamma+1)}{\Gamma(\gamma-\alpha+1)}(b-x)^{\gamma-\alpha} .
\end{aligned}
$$

Remark 2.7 Let $n-1<\alpha \leq n, n \in \mathbb{N}, 0 \leq \beta \leq 1, k=0,1, \ldots, n-1$, and $i=2,3, \ldots, n$. Then, we get

$$
\begin{aligned}
& \left(\mathcal{D}_{a^{+}}^{n-(n-\alpha)(1-\beta)-i, \beta}(t-a)^{k-(n-\alpha)(1-\beta)}\right)(x)=\frac{\Gamma(k-(n-\alpha)(1-\beta)+1)}{\Gamma(k+i-n+1)}(x-a)^{k+i-n}, \\
& \left(\mathcal{I}_{a^{+}}^{n-\alpha}(t-a)^{k-(n-\alpha)(1-\beta)}\right)(x)=\frac{\Gamma(k-(n-\alpha)(1-\beta)+1)}{\Gamma(k+\beta(n-\alpha)+1)}(x-a)^{k+\beta(n-\alpha)} .
\end{aligned}
$$

Note that these particular power rules will play crucial roles in what follows.

Here we begin the second part of this section, which includes a quick overview on the coincidence degree theory. For detailed discussions, we refer to Chapters IV and V of $[15,22]$, and [29]. 
Definition 2.8 Let $X$ and $Y$ be real normed spaces. A linear mapping $\mathcal{L}: \operatorname{dom} \mathcal{L} \subset X \rightarrow Y$ is called a Fredholm mapping if the following conditions hold:

(i) $\operatorname{ker} \mathcal{L}$ has a finite dimension,

(ii) $\operatorname{Im} \mathcal{L}$ is closed and has a finite codimension.

Let $\mathcal{L}$ be a Fredholm mapping. Then its index is given by

Ind $\mathcal{L}=\operatorname{dim} \operatorname{ker} \mathcal{L}-\operatorname{codim} \operatorname{Im} \mathcal{L}$.

Let $\mathcal{L}$ is a Fredholm mapping with index zero and suppose that there exist continuous projectors $P: X \rightarrow X$ and $Q: Y \rightarrow Y$ such that

$$
\operatorname{Im} P=\operatorname{ker} \mathcal{L}, \quad \operatorname{ker} Q=\operatorname{Im} \mathcal{L}, \quad X=\operatorname{ker} \mathcal{L} \oplus \operatorname{ker} P, \quad Y=\operatorname{Im} \mathcal{L} \oplus \operatorname{Im} Q
$$

It follows that the mapping

$$
\left.\mathcal{L}\right|_{\operatorname{dom} \mathcal{L} \cap \operatorname{ker} P}: \operatorname{dom} \mathcal{L} \cap \operatorname{ker} P \rightarrow \operatorname{Im} \mathcal{L}
$$

is invertible. We denote this inverse by $K_{P}: \operatorname{Im} \mathcal{L} \rightarrow \operatorname{dom} \mathcal{L} \cap \operatorname{ker} P$. The generalized inverse of $\mathcal{L}$ denoted by $K_{P, Q}: Z \rightarrow \operatorname{dom} \mathcal{L} \cap \operatorname{ker} P$ is defined by $K_{P, Q}=K_{P}(I-Q)$.

If $\mathcal{L}$ is a Fredholm mapping of index zero, then for every isomorphism $J: \operatorname{Im} Q \rightarrow \operatorname{ker} \mathcal{L}$, the mapping $J Q+K_{P, Q}: Z \rightarrow \operatorname{dom} \mathcal{L}$ is an isomorphism, and for every $u \in \operatorname{dom} \mathcal{L}$,

$$
\left(J Q+K_{P, Q}\right)^{-1} u=\left(\mathcal{L}+J^{-1} P\right) u .
$$

Definition 2.9 Let $\mathcal{L}: \operatorname{dom} \mathcal{L} \subset X \rightarrow Y$ be a Fredholm mapping, and let $E$ be a metric space. A mapping $\mathcal{N}: E \rightarrow Y$ is called $L$-compact on $E$ if $Q \mathcal{N}: E \rightarrow Y$ is continuous and $K_{P, Q} \mathcal{N}: E \rightarrow X$ is compact on $E$. In addition, we say that $\mathcal{N}$ is $L$-completely continuous if it is $L$-compact on every bounded subset $E \subset X$.

Theorem 2.10 Let $\Omega \subset X$ be open and bounded, let $\mathcal{L}$ be a Fredholm mapping of index zero, and let $\mathcal{N}$ be L-compact on $\bar{\Omega}$. Assume that the following conditions are satisfied:

(i) $\mathcal{L} u \neq \lambda \mathcal{N} u$ for every $(u, \lambda) \in((\operatorname{dom} \mathcal{L} \backslash \operatorname{ker} \mathcal{L}) \cap \partial \Omega) \times(0,1)$;

(ii) $\mathcal{N} u \notin \operatorname{Im} \mathcal{L}$ for every $u \in \operatorname{ker} \mathcal{L} \cap \partial \Omega$;

(iii) $\operatorname{deg}\left(\left.J Q \mathcal{N}\right|_{\text {ker } \mathcal{L} \cap \partial \Omega}, \Omega \cap \operatorname{ker} \mathcal{L}, 0\right) \neq 0$ with a continuous projector $Q: Y \rightarrow Y$ such that $\operatorname{ker} Q=\operatorname{Im} \mathcal{L}$ and $J: \operatorname{Im} Q \rightarrow \operatorname{ker} \mathcal{L}$ is an isomorphism.

Then the equation $\mathcal{L} u=\mathcal{N} u$ has at least one solution in $\operatorname{dom} \mathcal{L} \cap \bar{\Omega}$.

At the end of this part, we reveal why the Hilfer fractional coupled system (1.1)-(1.2) is of resonance category. Let us consider the homogeneous Hilfer fractional system

$$
\begin{cases}D_{a^{+}}^{\alpha, \beta} u(t)=0, & a<t<b, \\ D_{a^{+}}^{\alpha, \beta} v(t)=0, & a<t<b\end{cases}
$$

subject to the boundary conditions

$$
\begin{cases}D_{a^{+}}^{n-(n-\alpha)(1-\beta)-i, \beta} u(a)=0, & D_{a^{+}}^{n-(n-\alpha)(1-\beta)-1, \beta} u(a)=D_{a^{+}}^{n-(n-\alpha)(1-\beta)-1, \beta} u(b), \\ D_{a^{+}}^{n-(n-\alpha)(1-\beta)-i, \beta} v(a)=0, & D_{a^{+}}^{n-(n-\alpha)(1-\beta)-1, \beta} v(a)=D_{a^{+}}^{n-(n-\alpha)(1-\beta)-1, \beta} v(b),\end{cases}
$$


where $i=2,3, \ldots, n$. Having identity (2.10) in hand and imposing the boundary conditions

$$
D_{a^{+}}^{n-(n-\alpha)(1-\beta)-i, \beta} u(a)=0, \quad i=2,3, \ldots, n,
$$

it follows that $c_{k}=0, k=0,1, \ldots, n-3$, whereas the coefficients $c_{n-2}$ and $c_{n-1}$ are arbitrary. Now imposing the boundary condition

$$
D_{a^{+}}^{n-(n-\alpha)(1-\beta)-1, \beta} u(a)=D_{a^{+}}^{n-(n-\alpha)(1-\beta)-1, \beta} u(b)
$$

yields $c_{n-2}=0$. So, we can conclude that the homogeneous Hilfer fractional system (2.15)(2.16) has a nontrivial solution of the form

$$
(u(t), v(t))=\left(c_{n-1}(t-a)^{n-(n-\alpha)(1-\beta)-1}, c_{n-1}(t-a)^{n-(n-\alpha)(1-\beta)-1}\right) .
$$

Therefore our situation lies in the resonance category.

We finalize this section by presenting appropriate function spaces and their relevant norms. We first define the following Banach spaces:

$$
\begin{aligned}
& \mathcal{B}:=\left\{u \mid u, D_{a^{+}}^{n-(n-\alpha)(1-\beta)-i, \beta} u \in C[a, b], i=1,2, \ldots, n\right\}, \\
& \|u\|_{\mathcal{B}}:=\max \left\{\|u\|,\left\|D_{a^{+}}^{n-(n-\alpha)(1-\beta)-i, \beta} u\right\|, i=1,2, \ldots, n\right\}, \\
& \mathcal{E}:=\{y \mid y \in C[a, b]\}, \quad\|y\|:=\max _{t \in[a, b]}|y(t)| .
\end{aligned}
$$

Accordingly, the appropriate Banach spaces in this paper are given as follows:

$$
\begin{aligned}
& X:=\mathcal{B} \times \mathcal{B}, \quad\|(u, v)\|_{X}:=\max \left\{\|u\|_{\mathcal{B}},\|v\|_{\mathcal{B}}\right\}, \\
& Y:=\mathcal{E} \times \mathcal{E}, \quad\|(y, z)\|_{Y}:=\max \{\|y\|,\|z\|\} .
\end{aligned}
$$

\section{Main results}

In this section, we obtain some existence and uniqueness criteria for the Hilfer fractional resonant system (1.1)-(1.2). To this aim, as explained in the previous section, we are going to apply the coincidence degree theory. So, based on Theorem 2.10, we first transform the Hilfer system (1.1)-(1.2) into an adequate abstract equation such as $\mathcal{L}(u, v)=\mathcal{N}(u, v)$. Here we construct required elements of this transformation:

$$
\begin{aligned}
& \mathcal{L}(u, v):=\left(\mathcal{L}_{1} u, \mathcal{L}_{2} v\right), \quad\left\{\begin{array}{l}
\mathcal{L}_{1} u:=D_{a^{+}}^{\alpha, \beta} u, \\
\mathcal{L}_{2} v:=D_{a^{+}}^{\alpha, \beta} v,
\end{array}\right. \\
& \operatorname{dom} \mathcal{L}:=\left(\operatorname{dom} \mathcal{L}_{1}, \operatorname{dom} \mathcal{L}_{2}\right),
\end{aligned}
$$

where

$$
\begin{aligned}
\operatorname{dom} \mathcal{L}_{1}:= & \left\{u \in \mathcal{B} \mid D_{a^{+}}^{n-(n-\alpha)(1-\beta)-i, \beta} u(a)=0,\right. \\
& \left.D_{a^{+}}^{n-(n-\alpha)(1-\beta)-1, \beta} u(a)=D_{a^{+}}^{n-(n-\alpha)(1-\beta)-1, \beta} u(b), i \in \mathbb{N}_{2}^{n}\right\}, \\
\operatorname{dom} \mathcal{L}_{2}:= & \left\{v \in \mathcal{B} \mid D_{a^{+}}^{n-(n-\alpha)(1-\beta)-i, \beta} v(a)=0,\right. \\
& \left.D_{a^{+}}^{n-(n-\alpha)(1-\beta)-1, \beta} v(a)=D_{a^{+}}^{n-(n-\alpha)(1-\beta)-1, \beta} v(b), i \in \mathbb{N}_{2}^{n}\right\}
\end{aligned}
$$


Here we present the structure of the abstract nonlinearity $\mathcal{N}$ :

$$
\begin{aligned}
& \mathcal{N}(u, v):=\left(\mathcal{N}_{1} v, \mathcal{N}_{2} u\right) \\
& \left\{\begin{array}{l}
\mathcal{N}_{1} v:=\Phi\left(t, v, D_{a^{+}}^{1-(n-\alpha)(1-\beta), \beta} v, D_{a^{+}}^{2-(n-\alpha)(1-\beta), \beta} v, \ldots, D_{a^{+}}^{n-(n-\alpha)(1-\beta)-1, \beta} v\right), \\
\mathcal{N}_{2} u:=\Psi\left(t, u, D_{a^{+}}^{1-(n-\alpha)(1-\beta), \beta} u, D_{a^{+}}^{2-(n-\alpha)(1-\beta), \beta} u, \ldots, D_{a^{+}}^{n-(n-\alpha)(1-\beta)-1, \beta} u\right) .
\end{array}\right.
\end{aligned}
$$

Considering the setting (3.1)-(3.3), we come to the conclusion that the Hilfer resonant system (1.1)-(1.2) is equivalent to the abstract problem $\mathcal{L}(u, v)=\mathcal{N}(u, v)$. We further have to implement the basis of the coincidence degree theory, step by step as follows.

In the first step, we prove that the operator $\mathcal{L}(u, v)$ defined by (3.1) is a Fredholm operator of index 0 .

Lemma 3.1 The operator $\mathcal{L}: \operatorname{dom} \mathcal{L} \cap X \rightarrow Y$ is a Fredholm operator of index 0 .

Proof Thanks to identity (2.10) in Lemma 2.6, focussing on the operator $\mathcal{L}$ defined by (3.1) with $\operatorname{dom} \mathcal{L}$ defined by (3.3), we get that

$$
\operatorname{ker} \mathcal{L}=\left(c_{n-1}(t-a)^{n-(n-\alpha)(1-\beta)-1}, d_{n-1}(t-a)^{n-(n-\alpha)(1-\beta)-1}\right)
$$

which yields $\operatorname{ker} \mathcal{L} \cong \mathbb{R}^{2}$. Now let $(x, y) \in \operatorname{Im} \mathcal{L}$. So there is a pair $(u, v) \in \operatorname{dom} \mathcal{L}$ such that $\mathcal{L}(u, v)=(x, y)$, that is,

$$
\begin{aligned}
u(t)=I_{a^{+}}^{\alpha} & x(t)+c_{0}(t-a)^{-(n-\alpha)(1-\beta)}+c_{1}(t-a)^{1-(n-\alpha)(1-\beta)} \\
& +\cdots+c_{n-1}(t-a)^{n-(n-\alpha)(1-\beta)-1}, \\
v(t)= & I_{a^{+}}^{\alpha} y(t)+d_{0}(t-a)^{-(n-\alpha)(1-\beta)}+d_{1}(t-a)^{1-(n-\alpha)(1-\beta)} \\
& +\cdots+d_{n-1}(t-a)^{n-(n-\alpha)(1-\beta)-1} .
\end{aligned}
$$

According to the $\operatorname{dom} \mathcal{L}$, we conclude that $c_{i}=d_{i}=0, i=0,1, \ldots, n-2$. Thus we arrive at

$$
\begin{aligned}
& u(t)=I_{a^{+}}^{\alpha} x(t)+c_{n-1}(t-a)^{n-(n-\alpha)(1-\beta)-1}, \\
& v(t)=I_{a^{+}}^{\alpha} y(t)+d_{n-1}(t-a)^{n-(n-\alpha)(1-\beta)-1} .
\end{aligned}
$$

Equivalently, we have

$$
\begin{aligned}
& D_{a^{+}}^{n-(n-\alpha)(1-\beta)-1, \beta} u(t)=I_{a^{+}}^{1-\beta(n-\alpha)} x(t)+c_{n-1} \Gamma(n-(n-\alpha)(1-\beta)), \\
& D_{a^{+}}^{n-(n-\alpha)(1-\beta)-1, \beta} v(t)=I_{a^{+}}^{1-\beta(n-\alpha)} y(t)+d_{n-1} \Gamma(n-(n-\alpha)(1-\beta)) .
\end{aligned}
$$

Next, implementing the boundary conditions

$$
\begin{aligned}
& D_{a^{+}}^{n-(n-\alpha)(1-\beta)-1, \beta} u(a)=D_{a^{+}}^{n-(n-\alpha)(1-\beta)-1, \beta} u(b), \\
& D_{a^{+}}^{n-(n-\alpha)(1-\beta)-1, \beta} v(a)=D_{a^{+}}^{n-(n-\alpha)(1-\beta)-1, \beta} v(b)
\end{aligned}
$$

into equalities (3.5) gives us the structure of the $\operatorname{Im} \mathcal{L}$ as follows:

$$
\int_{a}^{b}(b-s)^{-\beta(n-\alpha)} x(s) d s=0, \quad \int_{a}^{b}(b-s)^{-\beta(n-\alpha)} y(s) d s=0 .
$$


In other words,

$$
\operatorname{Im} \mathcal{L}:=\left\{(x, y) \mid \int_{a}^{b}(b-s)^{-\beta(n-\alpha)} x(s) d s=0, \int_{a}^{b}(b-s)^{-\beta(n-\alpha)} y(s) d s=0\right\}
$$

To get the desired outcome, we define the operator $Q: Y \rightarrow Y$ with $Q(x, y)=\left(Q_{1} x, Q_{2} y\right)$, where

$$
\begin{aligned}
& Q_{1}: \mathcal{E} \rightarrow \mathcal{E}, Q_{1} x(t):=\frac{1-\beta(n-\alpha)}{(b-a)^{1-\beta(n-\alpha)}} \int_{a}^{b}(b-s)^{-\beta(n-\alpha)} x(s) d s, \\
& Q_{2}: \mathcal{E} \rightarrow \mathcal{E}, \quad Q_{2} y(t):=\frac{1-\beta(n-\alpha)}{(b-a)^{1-\beta(n-\alpha)}} \int_{a}^{b}(b-s)^{-\beta(n-\alpha)} y(s) d s .
\end{aligned}
$$

It is easy to check that $\operatorname{Im} \mathcal{L}=\operatorname{ker} Q$ and $Q_{i}^{2}=Q_{i}, i=1,2$. So, we deduce that $Q^{2}(u, v)=$ $Q(u, v)$. This is the expected opportunity to complete the proof. Since $(x, y)=(x, y)-$ $Q(x, y)+Q(x, y)$, we have $Y=\operatorname{Im} \mathcal{L}+\operatorname{Im} Q$. Furthermore $\operatorname{Im} \mathcal{L} \cap \operatorname{Im} Q=\{(0,0)\}$ leads us to the fact that $Y=\operatorname{Im} \mathcal{L} \oplus \operatorname{Im} Q$. Next, we define the operator $P: X \rightarrow X$ with $P(u, v)=\left(P_{1} u, P_{2} v\right)$, where

$$
\begin{array}{ll}
P_{1}: \mathcal{B} \rightarrow \mathcal{B}, & P_{1} u(t):=\frac{D_{a^{+}}^{n-(n-\alpha)(1-\beta)-1, \beta} u(a)}{\Gamma(n-(n-\alpha)(1-\beta))}(t-a)^{n-(n-\alpha)(1-\beta)-1}, \\
P_{2}: \mathcal{B} \rightarrow \mathcal{B}, \quad P_{2} v(t):=\frac{D_{a^{+}}^{n-(n-\alpha)(1-\beta)-1, \beta} v(a)}{\Gamma(n-(n-\alpha)(1-\beta))}(t-a)^{n-(n-\alpha)(1-\beta)-1} .
\end{array}
$$

The definitions of the operators $P_{i}, i=1,2$, immediately give us $P_{i}^{2}=P_{i}, i=1,2$, that is, $P^{2}(u, v)=P(u, v)$.

Definitions (3.9) and (3.10) imply that

$$
\operatorname{ker} P:=\left\{(u, v) \in X \mid D_{a^{+}}^{n-(n-\alpha)(1-\beta)-1, \beta} u(a)=0, D_{a^{+}}^{n-(n-\alpha)(1-\beta)-1, \beta} v(a)=0\right\} .
$$

Since $(u, v)=(u, v)-P(u, v)+P(u, v)$, we can derive that $X:=\operatorname{ker} P+\operatorname{ker} \mathcal{L}$. On the other hand, since $\operatorname{ker} P \cap \operatorname{ker} \mathcal{L}=\{(0,0)\}$, we have $X:=\operatorname{ker} P \oplus \operatorname{ker} \mathcal{L}$. So, according to Definition 2.8, and the fact that $\operatorname{ker} Q=\operatorname{Im} \mathcal{L}$, we conclude that

$$
\operatorname{dim} \operatorname{ker} \mathcal{L}:=\operatorname{codim} \operatorname{Im} \mathcal{L}=2
$$

Now the proof is complete.

In this position, we begin the second step proving the $L$-compactness of the operator $\mathcal{N}$ defined by (3.4). Let us define the operator $K_{P}: \operatorname{Im} \mathcal{L} \rightarrow \operatorname{dom} \mathcal{L} \cap \operatorname{ker} P$ by

$$
K_{P}(x, y):=\left(I_{a^{+}}^{\alpha} x, I_{a^{+}}^{\alpha} y\right)
$$

So we directly get that for each $(x, y) \in \operatorname{Im} \mathcal{L}$,

$$
\mathcal{L} K_{P}(x, y):=\left(D_{a^{+}}^{\alpha, \beta} I_{a^{+}}^{\alpha} x, D_{a^{+}}^{\alpha, \beta} I_{a^{+}}^{\alpha} y\right)=(x, y) .
$$


Next, let $(u, v) \in \operatorname{dom} \mathcal{L} \cap \operatorname{ker} P$. Therefore, in the identities

$$
\begin{aligned}
u(t)= & I_{a^{+}}^{\alpha} D_{a^{+}}^{\alpha, \beta} u(t)+c_{0}(t-a)^{-(n-\alpha)(1-\beta)}+c_{1}(t-a)^{1-(n-\alpha)(1-\beta)} \\
& +\cdots+c_{n-1}(t-a)^{n-(n-\alpha)(1-\beta)-1}, \\
v(t)= & I_{a^{+}}^{\alpha} D_{a^{+}}^{\alpha, \beta} v(t)+d_{0}(t-a)^{-(n-\alpha)(1-\beta)}+d_{1}(t-a)^{1-(n-\alpha)(1-\beta)} \\
& +\cdots+d_{n-1}(t-a)^{n-(n-\alpha)(1-\beta)-1},
\end{aligned}
$$

all the coefficients $c_{i}$ and $d_{i}$ vanish for $i=0,1, \ldots, n-1$, which yields

$$
K_{P} \mathcal{L}(u, v):=\left(I_{a^{+}}^{\alpha} D_{a^{+}}^{\alpha, \beta} u, I_{a^{+}}^{\alpha} D_{a^{+}}^{\alpha, \beta} v\right)=(u, v) .
$$

Finally, relying on (3.13) and (3.14), we conclude that $K_{P}=\left(\mathcal{L}_{\operatorname{dom} \mathcal{L} \cap \operatorname{ker} P}\right)^{-1}$.

Here we prove the $L$-compactness of the operator $\mathcal{N}$ in the following lemma.

Lemma 3.2 Let $\Omega$ be an open bounded subset of $X$ such that $\operatorname{dom} \mathcal{L} \cap \bar{\Omega} \neq \varnothing$. Then the operator $\mathcal{N}$ defined by (3.4) is L-compact.

Proof General assumption $\left(A_{2}\right)$ emphasizes that the nonlinearities $\Phi$ and $\Psi$ both are continuous, that is, the operator $\mathcal{N}$ is continuous. So, we immediately come to the conclusion that both operators $Q \mathcal{N}(\bar{\Omega})$ and $K_{P}(I-Q) \mathcal{N}(\bar{\Omega})$ are bounded. To prove the $L$-compactness of the operator $\mathcal{N}$, according to the Arzelà-Ascoli theorem, it just remains to prove the equicontinuity of the operator $K_{P, Q}:=K_{P}(I-Q) \mathcal{N}(\bar{\Omega})$. Recalling the continuity of $\Phi$ and $\Psi$ once again, we get that there exist positive constants $M_{i}, i=1,2$, such that for all $t \in[a, b]$ and $(u, v) \in \bar{\Omega}$, we have $|(I-Q) \mathcal{N} u(t)| \leq M_{1}$ and $|(I-Q) \mathcal{N} v(t)| \leq M_{2}$. Therefore, for each $a \leq t_{1}<t_{2} \leq b,(u, v) \in \bar{\Omega}$, and $i=1,2, \ldots, n-1$, we have

$$
\begin{aligned}
& \left|K_{P, Q} u\left(t_{2}\right)-K_{P, Q} u\left(t_{1}\right)\right| \\
& \quad:=\left|I_{a^{+}}^{\alpha} u\left(t_{2}\right)-I_{a^{+}}^{\alpha} u\left(t_{2}\right)\right| \\
& \quad=\frac{1}{\Gamma(\alpha)}\left|\int_{a}^{t_{2}}\left(t_{2}-s\right)^{\alpha-1}(I-Q) \mathcal{N} u(s) d s-\int_{a}^{t_{1}}\left(t_{1}-s\right)^{\alpha-1}(I-Q) \mathcal{N} u(s) d s\right| \\
& \quad \leq \frac{M_{1}}{\Gamma(\alpha)} \int_{a}^{t_{1}}\left\{\left(t_{2}-s\right)^{\alpha-1}-\left(t_{1}-s\right)^{\alpha-1}\right\} d s+\int_{t_{1}}^{t_{2}}\left(t_{2}-s\right)^{\alpha-1} d s \\
& =\frac{M_{1}}{\Gamma(\alpha+1)}\left\{\left(t_{2}-a\right)^{\alpha}-\left(t_{1}-a\right)^{\alpha}\right\}
\end{aligned}
$$

and, similarly,

$$
\left|K_{P, Q} v\left(t_{2}\right)-K_{P, Q} v\left(t_{1}\right)\right| \leq \frac{M_{2}}{\Gamma(\alpha+1)}\left\{\left(t_{2}-a\right)^{\alpha}-\left(t_{1}-a\right)^{\alpha}\right\} .
$$

In addition,

$$
\begin{aligned}
& \left|D_{a^{+}}^{n-(n-\alpha)(1-\beta)-i, \beta} K_{P, Q} u\left(t_{2}\right)-D_{a^{+}}^{n-(n-\alpha)(1-\beta)-i, \beta} K_{P, Q} u\left(t_{2}\right)\right| \\
& \quad:=\left|D_{a^{+}}^{n-(n-\alpha)(1-\beta)-i, \beta} I_{a^{+}}^{\alpha}(I-Q) \mathcal{N} u\left(t_{2}\right)-D_{a^{+}}^{n-(n-\alpha)(1-\beta)-i, \beta} I_{a^{+}}^{\alpha}(I-Q) \mathcal{N} u\left(t_{1}\right)\right| \\
& \quad=\left|I_{a^{+}}^{i-\beta(n-\alpha)}(I-Q) \mathcal{N} u\left(t_{2}\right)-I_{a^{+}}^{i-\beta(n-\alpha)}(I-Q) \mathcal{N} u\left(t_{1}\right)\right|
\end{aligned}
$$




$$
\begin{aligned}
= & \frac{1}{\Gamma(i-\beta(n-\alpha))} \\
& \times\left|\int_{a}^{t_{2}}\left(t_{2}-s\right)^{i-\beta(n-\alpha)-1}(I-Q) \mathcal{N} u(s) d s-\int_{a}^{t_{1}}\left(t_{1}-s\right)^{i-\beta(n-\alpha)-1}(I-Q) \mathcal{N} u(s) d s\right| \\
\leq & \frac{M_{1}}{\Gamma(i-\beta(n-\alpha))} \\
& \times \int_{a}^{t_{1}}\left\{\left(t_{2}-s\right)^{i-\beta(n-\alpha)-1}-\left(t_{1}-s\right)^{i-\beta(n-\alpha)-1}\right\} d s+\int_{t_{1}}^{t_{2}}\left(t_{2}-s\right)^{i-\beta(n-\alpha)-1} d s \\
= & \frac{M_{1}}{\Gamma(i-\beta(n-\alpha)+1)}\left\{\left(t_{2}-a\right)^{i-\beta(n-\alpha)}-\left(t_{1}-a\right)^{i-\beta(n-\alpha)}\right\}
\end{aligned}
$$

and, similarly,

$$
\begin{aligned}
& \left|D_{a^{+}}^{n-(n-\alpha)(1-\beta)-i, \beta} K_{P, Q} v\left(t_{2}\right)-D_{a^{+}}^{n-(n-\alpha)(1-\beta)-i, \beta} K_{P, Q} v\left(t_{2}\right)\right| \\
& \quad \leq \frac{M_{2}}{\Gamma(i-\beta(n-\alpha)+1)}\left\{\left(t_{2}-a\right)^{i-\beta(n-\alpha)}-\left(t_{1}-a\right)^{i-\beta(n-\alpha)}\right\} .
\end{aligned}
$$

At the and, because of the uniform continuity of the functions $\rho(t):=(t-a)^{\alpha}$ and $\rho_{i}(t):=(t-a)^{i-\beta(n-\alpha)}, i=1,2, \ldots, n-1$ on $[a, b]$, we can derive that $K_{P, Q}(\bar{\Omega}) \subset C[a, b]$ and $D_{a^{+}}^{n-(n-\alpha)(1-\beta)-i, \beta} K_{P, Q}(\bar{\Omega}) \subset C[a, b]$ are bounded and equicontinuous. This guarantees that the operator $K_{P, Q}:=K_{P}(I-Q) \mathcal{N}$ is compact on $\bar{\Omega}$, which yields the $L$-compactness of the operator $\mathcal{N}$.

Remark 3.3 Let $(u, v) \in X$. So,

$$
\|P(u, v)\|_{X}=\left\|\left(P_{1} u, P_{2} v\right)\right\|_{X}:=\max \left\{\left\|P_{1} u\right\|_{\mathcal{B}},\left\|P_{2} v\right\|_{\mathcal{B}}\right\} .
$$

Equivalently, for each $i=1,2, \ldots, n-1$, we have

$$
\begin{aligned}
\|P(u, v)\|_{X}= & \max \left\{\max \left\{\left\|P_{1} u\right\|_{\mathcal{B}},\left\|D_{a^{+}}^{n-(n-\alpha)(1-\beta)-i, \beta} P_{1} u\right\|_{\mathcal{B}}\right\},\right. \\
& \left.\max \left\{\left\|P_{2} v\right\|_{\mathcal{B}},\left\|D_{a^{+}}^{n-(n-\alpha)(1-\beta)-i, \beta} P_{2} v\right\|_{\mathcal{B}}\right\}\right\} .
\end{aligned}
$$

Now, using the power rule (2.11) and definitions (3.9) and (3.10), we conclude that

$$
\|P(u, v)\|_{X} \leq \Lambda_{1} \max \left\{\left|D_{a^{+}}^{n-(n-\alpha)(1-\beta)-1, \beta} u(a)\right|,\left|D_{a^{+}}^{n-(n-\alpha)(1-\beta)-1, \beta} v(a)\right|\right\},
$$

where

$$
\Lambda_{1}:=\frac{\max \left\{(b-a)^{n-(n-\alpha)(1-\beta)-1}, \frac{\Gamma(n-(n-\alpha)(1-\beta))}{(i-1) !}(b-a)^{i}, i=1,2, \ldots, n-1\right\}}{\Gamma(n-(n-\alpha)(1-\beta))} .
$$

Similarly, we have

$$
\left\|K_{P}(u, v)\right\|_{X} \leq \Lambda_{2} \max \left\{\|u\|_{\mathcal{B}},\|v\|_{\mathcal{B}}\right\}
$$

where

$$
\Lambda_{2}:=\max \left\{\frac{(b-a)^{\alpha}}{\Gamma(\alpha+1)}, \frac{(b-a)^{i-\beta(n-\alpha)}}{\Gamma(i-\beta(n-\alpha)+1)}, i=1,2, \ldots, n-1\right\} .
$$


Turning to Theorem 2.10, we have just been proved that the operators $\mathcal{L}$ defined by (3.1) and $\mathcal{N}$ defined by (3.4) are a Fredholm operator of index 0 and an $L$-compact operator, respectively. In what follows, we are going to identify conditions (i)-(iii) in this theorem. To this aim, we first consider the following hypotheses:

$\left(H_{1}\right)$ There exist positive real constants $d_{i}, i=1,2, b_{k}, c_{k}, \theta_{k}$, and $\lambda_{k}$ with $\theta_{k}, \lambda_{k} \in[0,1]$ for $k=1,2, \ldots, n$ such that for each $\left(x_{1}, x_{2}, \ldots, x_{n}\right) \in \mathbb{R}^{n}$,

$$
\begin{aligned}
& \left|\Phi\left(t, x_{1}, x_{2}, \ldots, x_{n}\right)\right| \leq d_{1}+\sum_{k=1}^{n} b_{k}\left|x_{k}\right|^{\theta_{k}}, \quad t \in[a, b] \\
& \left|\Psi\left(t, x_{1}, x_{2}, \ldots, x_{n}\right)\right| \leq d_{2}+\sum_{k=1}^{n} c_{k}\left|x_{k}\right|^{\lambda_{k}}, \quad t \in[a, b] .
\end{aligned}
$$

$\left(H_{2}\right)$ For a positive real constant $B$, such that for each $x_{i}, y_{i} \in \mathbb{R}, i=1,2, \ldots, n$, if $\min \left\{\left|x_{n}\right|,\left|y_{n}\right|\right\}>B$, then we have either

$$
x_{n} \cdot \Phi\left(t, y_{1}, y_{2}, \ldots, y_{n}\right)>0, \quad \text { or } \quad x_{n} \cdot \Phi\left(t, y_{1}, y_{2}, \ldots, y_{n}\right)<0, \quad t \in[a, b]
$$

or

$$
y_{n} \cdot \Phi\left(t, x_{1}, x_{2}, \ldots, x_{n}\right)>0, \quad \text { or } \quad y_{n} \cdot \Phi\left(t, x_{1}, x_{2}, \ldots, x_{n}\right)<0, \quad t \in[a, b] \text {. }
$$

$\left(H_{3}\right)$

$$
\begin{aligned}
& \left(\Lambda_{1} \Theta+\Lambda_{2}\right) \sum_{k=1}^{n} \xi_{k}<1, \quad \xi:=b, c, \\
& \Lambda_{1} \Theta \sum_{k=1}^{n} b_{k}+\Lambda_{2} \sum_{k=1}^{n} c_{k}<1, \\
& \Lambda_{1} \Theta \sum_{k=1}^{n} c_{k}+\Lambda_{2} \sum_{k=1}^{n} b_{k}<1,
\end{aligned}
$$

where $\Theta$ is given by $(3.28)$.

Here we define

$$
\begin{aligned}
& \Omega_{1}=\{(u, v) \in \operatorname{dom} \mathcal{L} \backslash \operatorname{ker} \mathcal{L} \mid \mathcal{L}(u, v)=\lambda \mathcal{N}(u, v), \lambda \in[0,1]\}, \\
& \Omega_{2}=\{(u, v) \in \operatorname{ker} \mathcal{L} \mid \mathcal{N}(u, v) \in \operatorname{Im} \mathcal{L}\}, \\
& \Omega_{3}=\{(u, v) \in \operatorname{ker} \mathcal{L} \mid \lambda(u, v)+(1-\lambda) Q \mathcal{N}(u, v)=(0,0), \lambda \in[0,1]\}, \\
& \Omega_{3}^{\prime}=\{(u, v) \in \operatorname{ker} \mathcal{L} \mid-\lambda(u, v)+(1-\lambda) Q \mathcal{N}(u, v)=(0,0), \lambda \in[0,1]\} .
\end{aligned}
$$

Indeed, if we prove that the subsets $\overline{\Omega_{i}} \subset X, i=1,2,3$, and $\overline{\Omega_{3}^{\prime}} \subset X$ are bounded, then relying on Theorem 2.10 , we can directly conclude the existence of at least one solution for the Hilfer fractional resonant system (1.1)-(1.2). So, we start to prove the boundedness of these sets.

Lemma 3.4 $\overline{\Omega_{1}}$ defined by (3.24a) is bounded subset of $X$. 
Proof For each $(u, v) \in \overline{\Omega_{1}},(u, v) \notin \operatorname{ker} \mathcal{L}$. So, $\lambda \neq 0$. Also, for each $(u, v) \in \overline{\Omega_{1}}$, we have $\mathcal{L}(u, v)=\lambda \mathcal{N}(u, v) \in \operatorname{ker} Q$, that is,

$$
\begin{aligned}
& \frac{\lambda(1-\beta(n-\alpha))}{(b-a)^{1-\beta(n-\alpha)}} \\
& \quad \times \int_{a}^{b}(b-s)^{-\beta(n-\alpha)} \Phi\left(s, v, D_{a^{+}}^{1-(n-\alpha)(1-\beta), \beta} v, \ldots, D_{a^{+}}^{n-(n-\alpha)(1-\beta)-1, \beta} v\right) d s=0, \\
& \frac{\lambda(1-\beta(n-\alpha))}{(b-a)^{1-\beta(n-\alpha)}} \\
& \quad \times \int_{a}^{b}(b-s)^{-\beta(n-\alpha)} \Psi\left(s, u, D_{a^{+}}^{1-(n-\alpha)(1-\beta), \beta} u, \ldots, D_{a^{+}}^{n-(n-\alpha)(1-\beta)-1, \beta} u\right) d s=0 .
\end{aligned}
$$

Thus there are $t_{0}, t_{1} \in[a, b]$ such that

$$
\begin{aligned}
& \Phi\left(t_{1}, v, D_{a^{+}}^{1-(n-\alpha)(1-\beta), \beta} v, D_{a^{+}}^{2-(n-\alpha)(1-\beta), \beta} v, \ldots, D_{a^{+}}^{n-(n-\alpha)(1-\beta)-1, \beta} v\right)=0 \\
& \Psi\left(t_{0}, u, D_{a^{+}}^{1-(n-\alpha)(1-\beta), \beta} u, D_{a^{+}}^{2-(n-\alpha)(1-\beta), \beta} u, \ldots, D_{a^{+}}^{n-(n-\alpha)(1-\beta)-1, \beta} u\right)=0
\end{aligned}
$$

In viewpoint of hypothesis $\left(H_{2}\right)$, equalities (3.25) imply that

$$
D_{a^{+}}^{n-(n-\alpha)(1-\beta)-1, \beta} u\left(t_{0}\right) \leq B, \quad D_{a^{+}}^{n-(n-\alpha)(1-\beta)-1, \beta} v\left(t_{1}\right) \leq B .
$$

For $(u, v) \in \overline{\Omega_{1}}$, we have that $\mathcal{L}(u, v)=\lambda \mathcal{N}(u, v)$, that is,

$$
D_{a^{+}}^{\alpha, \beta} u(t)=\lambda \mathcal{N}_{1} v(t), \quad D_{a^{+}}^{\alpha, \beta} \nu(t)=\lambda \mathcal{N}_{2} u(t)
$$

So, we have

$$
\begin{aligned}
& u(t)=\lambda I_{a^{+}}^{\alpha} \mathcal{N}_{1} v(t)+\sum_{k=0}^{n-1} \frac{(t-a)^{k-(n-\alpha)(1-\beta)}}{\Gamma(k-(n-\alpha)(1-\beta)+1)} D_{a^{+}}^{k-(n-\alpha)(1-\beta)} u(a), \\
& v(t)=\lambda I_{a^{+}}^{\alpha} \mathcal{N}_{2} u(t)+\sum_{k=0}^{n-1} \frac{(t-a)^{k-(n-\alpha)(1-\beta)}}{\Gamma(k-(n-\alpha)(1-\beta)+1)} D_{a^{+}}^{k-(n-\alpha)(1-\beta)} v(a) .
\end{aligned}
$$

Now taking $D_{a^{+}}^{n-(n-\alpha)(1-\beta)-1, \beta}$ on both sides of these equalities, we get

$$
\begin{aligned}
& D_{a^{+}}^{n-(n-\alpha)(1-\beta)-1, \beta} u(t)=\lambda I_{a^{+}}^{1-\beta(n-\alpha)} \mathcal{N}_{1} v(t)+D_{a^{+}}^{n-(n-\alpha)(1-\beta)-1, \beta} u(a), \\
& D_{a^{+}}^{n-(n-\alpha)(1-\beta)-1, \beta} v(t)=\lambda I_{a^{+}}^{1-\beta(n-\alpha)} \mathcal{N}_{2} u(t)+D_{a^{+}}^{n-(n-\alpha)(1-\beta)-1, \beta} v(a)
\end{aligned}
$$

Setting $t=t_{0}$ in the first equality and $t=t_{1}$ in the second equality gives us

$$
\begin{aligned}
& \left|D_{a^{+}}^{n-(n-\alpha)(1-\beta)-1, \beta} u(a)\right| \leq B+\lambda \frac{(b-a)^{1-\beta(n-\alpha)}}{\Gamma(2-\beta(n-\alpha))}\|\Phi\|, \\
& \left|D_{a^{+}}^{n-(n-\alpha)(1-\beta)-1, \beta} v(a)\right| \leq B+\lambda \frac{(b-a)^{1-\beta(n-\alpha)}}{\Gamma(2-\beta(n-\alpha))}\|\Psi\| .
\end{aligned}
$$


Applying hypothesis $\left(H_{1}\right)$ to these inequalities, we get

$$
\begin{aligned}
& \left|D_{a^{+}}^{n-(n-\alpha)(1-\beta)-1, \beta} u(a)\right| \leq B+\lambda \Theta\left(d_{1}+b_{1}\|v\|^{\theta_{1}}+\sum_{k=2}^{n} b_{k}\left\|D_{a^{+}}^{k-(n-\alpha)(1-\beta)-1, \beta} v\right\|^{\theta_{k}}\right), \\
& \left|D_{a^{+}}^{n-(n-\alpha)(1-\beta)-1, \beta} v(a)\right| \leq B+\lambda \Theta\left(d_{2}+c_{1}\|u\|^{\lambda_{1}}+\sum_{k=2}^{n} c_{k}\left\|D_{a^{+}}^{k-(n-\alpha)(1-\beta)-1, \beta} u\right\|^{\lambda_{k}}\right),
\end{aligned}
$$

where

$$
\Theta:=\frac{(b-a)^{1-\beta(n-\alpha)}}{\Gamma(2-\beta(n-\alpha))} .
$$

Let $(u, v) \in \Omega_{1}$. Then $(u, v) \in \operatorname{dom} \mathcal{L} \backslash \operatorname{ker} \mathcal{L}$. Since $P^{2}=P,(I-P)(u, v) \in \operatorname{dom} \mathcal{L} \cap \operatorname{ker} P$ and $\mathcal{L} P(u, v)=(0,0)$. So, by inequality (3.16) it follows that

$$
\begin{aligned}
\|(I-P)(u, v)\|_{X} & =\left\|K_{P} \mathcal{L}(I-P)(u, v)\right\|_{X}=\left\|K_{P}\left(\mathcal{L}_{1} u, \mathcal{L}_{2} v\right)\right\|_{X} \\
& =\left\|\left(I_{a^{+}}^{\alpha} \mathcal{L}_{1} u, I_{a^{+}}^{\alpha} \mathcal{L}_{2} v\right)\right\|_{X} \leq \lambda \Lambda_{2} \max \left\{\left\|\mathcal{N}_{1} v\right\|_{\mathcal{B}},\left\|\mathcal{N}_{2} u\right\|_{\mathcal{B}}\right\} \\
& \leq \Lambda_{2} \max \left\{\left\|\mathcal{N}_{1} v\right\|_{\mathcal{B}},\left\|\mathcal{N}_{2} u\right\|_{\mathcal{B}}\right\} .
\end{aligned}
$$

Accordingly, based on Remark 3.3 and (3.29), we have

$$
\begin{aligned}
\|(u, v)\|_{X}= & \|P(u, v)+(I-P)(u, v)\|_{X} \leq\|P(u, v)\|_{X}+\|(I-P)(u, v)\|_{X} \\
\leq & \max \left\{\left\{\Lambda_{1}\left|D_{a^{+}}^{n-(n-\alpha)(1-\beta)-1, \beta} u(a)\right|+\Lambda_{2}\left\|\mathcal{N}_{1} v\right\|_{\mathcal{B}}\right\},\right. \\
& \left\{\Lambda_{1}\left|D_{a^{+}}^{n-(n-\alpha)(1-\beta)-1, \beta} v(a)\right|+\Lambda_{2}\left\|\mathcal{N}_{2} u\right\|_{\mathcal{B}}\right\}, \\
& \left\{\Lambda_{1}\left|D_{a^{+}}^{n-(n-\alpha)(1-\beta)-1, \beta} u(a)\right|+\Lambda_{2}\left\|\mathcal{N}_{2} u\right\|_{\mathcal{B}}\right\}, \\
& \left.\left\{\Lambda_{1}\left|D_{a^{+}}^{n-(n-\alpha)(1-\beta)-1, \beta} v(a)\right|+\Lambda_{2}\left\|\mathcal{N}_{1} v\right\|_{\mathcal{B}}\right\}\right\} .
\end{aligned}
$$

To complete the proof, since the right-hand side of inequality (3.30) depends on four cases, we will divide our boundedness estimation into four cases as follows:

Case i.

$$
\|(u, v)\|_{X} \leq \Lambda_{1}\left|D_{a^{+}}^{n-(n-\alpha)(1-\beta)-1, \beta} u(a)\right|+\Lambda_{2}\left\|\mathcal{N}_{1} v\right\|_{\mathcal{B}} .
$$

So, properties (3.17) and (3.18) in hypothesis $\left(H_{1}\right)$ and (3.26)-(3.28) yield

$$
\|(u, v)\|_{X} \leq \Lambda_{1} B+\left(\Lambda_{1} \Theta+\Lambda_{2}\right)\left(d_{1}+b_{1}\|v\|_{\mathcal{B}}^{\theta_{1}}+\sum_{k=2}^{n} b_{k}\left\|D_{a^{+}}^{k-(n-\alpha)(1-\beta)-1} v\right\|_{\mathcal{B}}^{\theta_{k}}\right) .
$$

Case ii.

$$
\|(u, v)\|_{X} \leq \Lambda_{1}\left|D_{a^{+}}^{n-(n-\alpha)(1-\beta)-1, \beta} v(a)\right|+\Lambda_{2}\left\|\mathcal{N}_{2} u\right\|_{\mathcal{B}}
$$

The resources of case i imply that

$$
\|(u, v)\|_{X} \leq \Lambda_{1} B+\left(\Lambda_{1} \Theta+\Lambda_{2}\right)\left(d_{2}+c_{1}\|u\|_{\mathcal{B}}^{\lambda_{1}}+\sum_{k=2}^{n} c_{k}\left\|D_{a^{+}}^{k-(n-\alpha)(1-\beta)-1} u\right\|_{\mathcal{B}}^{\lambda_{k}}\right) .
$$


Case iii.

$$
\|(u, v)\|_{X} \leq \Lambda_{1}\left|D_{a^{+}}^{n-(n-\alpha)(1-\beta)-1, \beta} u(a)\right|+\Lambda_{2}\left\|\mathcal{N}_{2} u\right\|_{\mathcal{B}} .
$$

We have

$$
\begin{aligned}
\|(u, v)\|_{X} \leq & \Lambda_{1} \Theta\left\{B+\left(d_{1}+b_{1}\|v\|_{\mathcal{B}}^{\theta_{1}}+\sum_{k=2}^{n} b_{k}\left\|D_{a^{+}}^{k-(n-\alpha)(1-\beta)-1} v\right\|_{\mathcal{B}}^{\theta_{k}}\right)\right\} \\
& +\Lambda_{2}\left(d_{2}+c_{1}\|u\|_{\mathcal{B}}^{\lambda_{1}}+\sum_{k=2}^{n} c_{k}\left\|D_{a^{+}}^{k-(n-\alpha)(1-\beta)-1} u\right\|_{\mathcal{B}}^{\lambda_{k}}\right) .
\end{aligned}
$$

Case iv.

$$
\|(u, v)\|_{X} \leq \Lambda_{1}\left|D_{a^{+}}^{n-(n-\alpha)(1-\beta)-1, \beta} v(a)\right|+\Lambda_{2}\left\|\mathcal{N}_{1} v\right\|_{\mathcal{B}} .
$$

We get that

$$
\begin{aligned}
\|(u, v)\|_{X} \leq & \Lambda_{1} \Theta\left\{B+\left(d_{2}+c_{1}\|u\|_{\mathcal{B}}^{\lambda_{1}}+\sum_{k=2}^{n} c_{k}\left\|D_{a^{+}}^{k-(n-\alpha)(1-\beta)-1} u\right\|_{\mathcal{B}}^{\lambda_{k}}\right)\right\} \\
& +\Lambda_{2}\left(d_{1}+b_{1}\|v\|_{\mathcal{B}}^{\theta_{1}}+\sum_{k=2}^{n} b_{k}\left\|D_{a^{+}}^{k-(n-\alpha)(1-\beta)-1} v\right\|_{\mathcal{B}}^{\theta_{k}}\right) .
\end{aligned}
$$

Some simple manipulations on inequalities (3.32), (3.34), (3.36), and (3.38), help us to reach the following results.

(i)

$$
\|(u, v)\|_{X} \leq \frac{\Lambda_{1} B+d_{1}\left(\Lambda_{1} \Theta+\Lambda_{2}\right)}{1-\left(\Lambda_{1} \Theta+\Lambda_{2}\right) \sum_{k=1}^{n} b_{k}} .
$$

(ii)

$$
\|(u, v)\|_{X} \leq \frac{\Lambda_{1} B+d_{2}\left(\Lambda_{1} \Theta+\Lambda_{2}\right)}{1-\left(\Lambda_{1} \Theta+\Lambda_{2}\right) \sum_{k=1}^{n} c_{k}} .
$$

(iii)

$$
\|(u, v)\|_{X} \leq \frac{\Lambda_{1} B+\left(\Lambda_{1} \Theta d_{1}+\Lambda_{2} d_{2}\right)}{1-\left\{\Lambda_{1} \Theta \sum_{k=1}^{n} b_{k}+\Lambda_{2} \sum_{i=k}^{n} c_{k}\right\}} .
$$

(iv)

$$
\|(u, v)\|_{X} \leq \frac{\Lambda_{1} B+\left(\Lambda_{1} \Theta d_{2}+\Lambda_{2} d_{1}\right)}{1-\left\{\Lambda_{1} \Theta \sum_{k=1}^{n} c_{k}+\Lambda_{2} \sum_{k=1}^{n} b_{k}\right\}} .
$$

Inequalities (3.39)-(3.42) ensure that $\overline{\Omega_{1}} \subset X$ is bounded.

Lemma $3.5 \overline{\Omega_{2}}$ defined by (3.24b) is a bounded subset of $X$. 
Proof Let $(u, v) \in \overline{\Omega_{2}}$. Then $u(t):=c_{n-1}(t-a)^{n-(n-\alpha)(1-\beta)-1}$ and $v(t):=d_{n-1}(t-a)^{n-(n-\alpha)(1-\beta)-1}$. On the other hand,

$$
\mathcal{N}(u, v)=\left(\mathcal{N}_{1} v, \mathcal{N}_{2} u\right) \in \operatorname{Im} \mathcal{L}=\operatorname{ker} Q
$$

So, we have

$$
\begin{aligned}
& \int_{a}^{b}(b-s)^{-\beta(n-\alpha)} \\
& \quad \times \Phi\left(s, c_{n-1}(s-a)^{n-(n-\alpha)(1-\beta)-1}, c_{n-1} D_{a^{+}}^{1-(n-\alpha)(1-\beta)}(s-a)^{n-(n-\alpha)(1-\beta)-1}\right. \\
& \left.\quad \ldots, c_{n-1} D_{a^{+}}^{n-(n-\alpha)(1-\beta)-1}(s-a)^{n-(n-\alpha)(1-\beta)-1}\right) d s=0
\end{aligned}
$$

and

$$
\begin{aligned}
& \int_{a}^{b}(b-s)^{-\beta(n-\alpha)} \\
& \quad \times \Psi\left(s, d_{n-1}(s-a)^{n-(n-\alpha)(1-\beta)-1}, d_{n-1} D_{a^{+}}^{1-(n-\alpha)(1-\beta)}(s-a)^{n-(n-\alpha)(1-\beta)-1},\right. \\
& \left.\quad \ldots, d_{n-1} D_{a^{+}}^{n-(n-\alpha)(1-\beta)-1}(s-a)^{n-(n-\alpha)(1-\beta)-1}\right) d s=0 .
\end{aligned}
$$

Now, according to hypothesis $\left(\mathrm{H}_{2}\right)$, we come to the conclusion that

$$
\left|c_{n-1}\right|,\left|d_{n-1}\right| \leq \frac{B}{\Gamma(n-(n-\alpha)(1-\beta))},
$$

that is, $\overline{\Omega_{2}}$ is a bounded subset of $X$.

The next case deals with the boundedness of the subset $\overline{\Omega_{3}}$.

Lemma $3.6 \overline{\Omega_{3}}$ defined by $(3.24 \mathrm{c})$ is a bounded subset of $X$.

Proof Our proof basically depends on hypothesis $\left(H_{2}\right)$. Let $(u, v) \in \overline{\Omega_{3}}$. Thus $(u, v) \in \operatorname{ker} \mathcal{L}$, that is,

$$
(u, v)=\left(c_{n-1}(t-a)^{n-(n-\alpha)(1-\beta)-1}, d_{n-1}(t-a)^{n-(n-\alpha)(1-\beta)-1}\right) .
$$

On the other hand, since $\operatorname{ker} \mathcal{L}=\operatorname{Im} Q$, from $\lambda(u, v)-(1-\lambda) Q \mathcal{N}(u, v)=(0,0)$ we deduce that

$$
\begin{aligned}
& \lambda c_{n-1}(t-a)^{n-(n-\alpha)(1-\beta)-1}+(1-\lambda) \int_{a}^{b}(b-s)^{-\beta(n-\alpha)} \\
& \quad \times \Phi\left(s, c_{n-1}(s-a)^{n-(n-\alpha)(1-\beta)-1}, c_{n-1} D_{a^{+}}^{1-(n-\alpha)(1-\beta)}(s-a)^{n-(n-\alpha)(1-\beta)-1},\right. \\
& \left.\quad \ldots, c_{n-1} D_{a^{+}}^{n-(n-\alpha)(1-\beta)-1}(s-a)^{n-(n-\alpha)(1-\beta)-1}\right) d s=0
\end{aligned}
$$


and

$$
\begin{aligned}
& \lambda d_{n-1}(t-a)^{n-(n-\alpha)(1-\beta)-1}+(1-\lambda) \int_{a}^{b}(b-s)^{-\beta(n-\alpha)} \\
& \quad \times \Psi\left(s, d_{n-1}(s-a)^{n-(n-\alpha)(1-\beta)-1}, d_{n-1} D_{a^{+}}^{1-(n-\alpha)(1-\beta)}(s-a)^{n-(n-\alpha)(1-\beta)-1},\right. \\
& \left.\quad \ldots, d_{n-1} D_{a^{+}}^{n-(n-\alpha)(1-\beta)-1}(s-a)^{n-(n-\alpha)(1-\beta)-1}\right) d s=0 .
\end{aligned}
$$

If in equalities (3.45) and (3.46), we take $\lambda=0$, then the proof reduces to the proof of Lemma 3.5. So, let $\lambda \in(0,1]$. Similarly to the previous case, this case gives us the desired outcome. The assumption $\left|c_{n-1}\right|,\left|d_{n-1}\right|>B$, together with the hypothesis $\left(H_{2}\right)$, leads us to

$$
\begin{aligned}
& \lambda c_{n-1}^{2}(t-a)^{2(n-(n-\alpha)(1-\beta)-1)}+(1-\lambda) c_{n-1} \int_{a}^{b}(b-s)^{-\beta(n-\alpha)}(s-a)^{n-(n-\alpha)(1-\beta)-1} \\
& \quad \times \Phi\left(s, c_{n-1}(s-a)^{n-(n-\alpha)(1-\beta)-1}, c_{n-1} D_{a^{+}}^{1-(n-\alpha)(1-\beta)}(s-a)^{n-(n-\alpha)(1-\beta)-1}\right. \\
& \left.\quad \ldots, c_{n-1} D_{a^{+}}^{n-(n-\alpha)(1-\beta)-1}(s-a)^{n-(n-\alpha)(1-\beta)-1}\right) d s>0
\end{aligned}
$$

and

$$
\begin{aligned}
& \lambda d_{n-1}^{2}(t-a)^{2(n-(n-\alpha)(1-\beta)-1)}+(1-\lambda) d_{n-1} \int_{a}^{b}(b-s)^{-\beta(n-\alpha)}(s-a)^{n-(n-\alpha)(1-\beta)-1} \\
& \times \Psi\left(s, d_{n-1}(s-a)^{n-(n-\alpha)(1-\beta)-1}, d_{n-1} D_{a^{+}}^{1-(n-\alpha)(1-\beta)}(s-a)^{n-(n-\alpha)(1-\beta)-1},\right. \\
& \left.\ldots, d_{n-1} D_{a^{+}}^{n-(n-\alpha)(1-\beta)-1}(s-a)^{n-(n-\alpha)(1-\beta)-1}\right) d s>0,
\end{aligned}
$$

which contradicts equalities (3.45) and (3.46). This completes the proof.

Concentrating on the second parts of hypothesis $\left(\mathrm{H}_{2}\right)$, that is, the right-hand side inequalities in (3.19) and (3.20), helps us to prove boundedness of $\overline{\Omega_{3}^{\prime}} \subset X$. So, we present this result without proof in the next lemma.

Lemma $3.7 \overline{\Omega_{3}^{\prime}}$ defined by (3.24d) is a bounded subset of $X$.

Since all conditions of Theorem 2.10 are satisfied in the frame of Lemmas 3.1, 3.2, and (3.4)-(3.7), we are ready to prove the existence of at least one solution of the Hilfer fractional resonant system (1.1)-(1.2) as follows.

Theorem 3.8 Assume that hypotheses $\left(H_{1}\right)-\left(H_{3}\right)$ are satisfied. Then the Hilfer fractional resonant system (1.1)-(1.2) has at least one solution in $X$.

Proof Consider $\Omega \supset \bigcup_{i=1}^{3} \overline{\Omega_{i}} \cup\{0\}$ (or $\Omega \supset \bigcup_{i=1}^{2} \overline{\Omega_{i}} \cup \Omega_{3}^{\prime} \cup\{0\}$ ) as a bounded open subset of $X$. According to the Lemma 3.2, we know that $\mathcal{N}$ is an $L$-compact operator on $\Omega$. Besides, via Lemmas 3.4-3.7, we get the following:

- $\mathcal{L}(u, v)=\lambda \mathcal{N}(u, v)$ for every $((u, v), \lambda) \in[\operatorname{dom} \mathcal{L} \backslash \operatorname{ker} \mathcal{L} \cap \partial \Omega] \times(0,1)$.

$-\mathcal{N}(u, v) \notin \operatorname{Im} \mathcal{L}$ for every $(u, v) \in \operatorname{ker} \mathcal{L} \cap \partial \Omega$.

So, it just remains to prove that

$\operatorname{deg}\left(\left.J Q \mathcal{N}\right|_{\operatorname{ker} \mathcal{L}}, \Omega \cap \operatorname{ker} \mathcal{L}, 0\right) \neq 0$. 
To prove this, we define the homotopy

$$
H((u, v), \lambda)= \pm \lambda \operatorname{Id}(u, v)+(1-\lambda) J Q \mathcal{N}(u, v)
$$

According to the degree property of invariance under a homotopy, if $u \in \operatorname{ker} \mathcal{L} \cap \partial \Omega$, then

$$
\begin{gathered}
\operatorname{deg}\left(\left.J Q \mathcal{N}\right|_{\operatorname{ker} \mathcal{L}}, \Omega \cap \operatorname{ker} \mathcal{L}, 0\right) \\
=\operatorname{deg}(H(\cdot, 0), \Omega \cap \operatorname{ker} \mathcal{L}, 0) \\
=\operatorname{deg}(H(\cdot, 1), \Omega \cap \operatorname{ker} \mathcal{L}, 0) \\
=\operatorname{deg}( \pm \operatorname{Id}, \Omega \cap \operatorname{ker} \mathcal{L}, 0) \neq 0 .
\end{gathered}
$$

Therefore, since assumption (iii) in Theorem 2.10 is satisfied, we conclude that the Hilfer fractional resonant system (1.1)-(1.2) has at least one solution in $X$.

As explained before, our investigation is divided into the couple of stages including the existence and uniqueness of solutions for the Hilfer fractional resonant system (1.1)-(1.2). So far, the existence of at least one solution is proved. So, in what follows, we state and prove a uniqueness criterion for the solutions of the coupled system (1.1)-(1.2). To do this, we first need the following hypotheses.

$\left(H_{1}^{\prime}\right)$ There exist positive constants $\left(\mu_{i}, \nu_{i}\right) \in \mathbb{R}^{2}, i=1, \ldots, n$, and $\left(\eta_{i}, \zeta_{i}\right) \in[0,1]^{2}, i=$ $1,2, \ldots, n$, such that for all $\left(\left(x_{i}\right)_{1}^{n},\left(y_{i}\right)_{1}^{n}\right) \in \mathbb{R}^{n} \times \mathbb{R}^{n}$,

$$
\left|\Phi\left(t, x_{1}, x_{2}, \ldots, x_{n}\right)-\Phi\left(t, y_{1}, y_{2}, \ldots, y_{n}\right)\right| \leq \sum_{i=1}^{n} \mu_{i}\left|x_{i}-y_{i}\right|^{\eta_{i}}
$$

and

$$
\left|\Psi\left(t, x_{1}, x_{2}, \ldots, x_{n}\right)-\Psi\left(t, y_{1}, y_{2}, \ldots, y_{n}\right)\right| \leq \sum_{i=1}^{n} v_{i}\left|x_{i}-y_{i}\right|^{\zeta_{i}}
$$

$\left(H_{2}^{\prime}\right)$ There exist positive constants $\left(k_{i}, l_{i}\right) \in \mathbb{R}^{2}, i=1, \ldots, n$, such that for all $\left(\left(x_{i}\right)_{1}^{n},\left(y_{i}\right)_{1}^{n}\right) \in$ $\mathbb{R}^{n} \times \mathbb{R}^{n}$

$$
\left|\Phi\left(t, x_{1}, x_{2}, \ldots, x_{n}\right)-\Phi\left(t, y_{1}, y_{2}, \ldots, y_{n}\right)\right| \geq k_{n}\left|x_{n}-y_{n}\right|-\sum_{i=1}^{n-1} k_{i}\left|x_{i}-y_{i}\right|
$$

and

$$
\left|\Psi\left(t, x_{1}, x_{2}, \ldots, x_{n}\right)-\Psi\left(t, y_{1}, y_{2}, \ldots, y_{n}\right)\right| \geq l_{n}\left|x_{n}-y_{n}\right|-\sum_{i=1}^{n-1} l_{i}\left|x_{i}-y_{i}\right| .
$$

$\left(H_{3}^{\prime}\right)$

$$
\Lambda_{1}\left\{\sum_{i=1}^{n-1} \frac{l_{i}}{l_{n}}+\Theta \sum_{i=1}^{n} \mu_{i}\right\}+\Lambda_{2} \sum_{i=1}^{n} b_{i}>1
$$




$$
\begin{aligned}
& \Lambda_{1}\left\{\sum_{i=1}^{n-1} \frac{k_{i}}{k_{n}}+\Theta \sum_{i=1}^{n} \nu_{i}\right\}+\Lambda_{2} \sum_{i=1}^{n} c_{i}>1, \\
& \Lambda_{1}\left\{\sum_{i=1}^{n-1} \frac{l_{i}}{l_{n}}+\Theta \sum_{i=1}^{n} \mu_{i}\right\}+\Lambda_{2} \sum_{i=1}^{n} c_{i}>1, \\
& \Lambda_{1}\left\{\sum_{i=1}^{n-1} \frac{k_{i}}{k_{n}}+\Theta \sum_{i=1}^{n} \nu_{i}\right\}+\Lambda_{2} \sum_{i=1}^{n} b_{i}>1 .
\end{aligned}
$$

Theorem 3.9 Assume that hypotheses $\left(H_{2}\right)-\left(H_{3}\right)$ and $\left(H_{1}^{\prime}\right)-\left(H_{3}^{\prime}\right)$ are satisfied. Then the Hilfer fractional resonant system (1.1)-(1.2) has a unique solution on $X$.

Proof To prove the uniqueness of solutions, we first will prove the existence of solutions. Since hypotheses $\left(H_{2}\right)-\left(H_{3}\right)$ are satisfied, we just need to check hypothesis $\left(H_{1}\right)$. To do this, for each $i=1,2, \ldots, n$, assume that $y_{i}=0$. Also suppose that

$$
d_{1}:=\Phi(t, \underbrace{0,0, \ldots, 0}_{n-1 \text { tupled }}), \quad d_{2}:=\Psi(t, \underbrace{0,0, \ldots, 0}_{n-1 \text { tupled }}) .
$$

In this case, hypothesis $\left(H_{1}^{\prime}\right)$ reduces to hypothesis $\left(H_{1}\right)$. Having the hypotheses $\left(H_{1}\right)-$ $\left(H_{3}\right)$ in hand, Theorem 3.8 guarantees the existence of at least one solution for the Hilfer fractional resonant system (1.1)-(1.2). Now it is time to prove the uniqueness of solutions. In the traditional way, we consider the solutions $\left(u_{k}, v_{k}\right) \in X, k=1,2$, of the coupled system (1.1)-(1.2) and will prove that $u_{1}=u_{2}$ and $v_{1}=v_{2}$. As supposed, for $k=1,2$,

$$
\begin{aligned}
& D_{a^{+}}^{\alpha, \beta} u_{k}(t)=\Phi\left(t, v_{k}, D_{a^{+}}^{1-(n-\alpha)(1-\beta), \beta} v_{k}, D_{a^{+}}^{2-(n-\alpha)(1-\beta), \beta} v_{k}, \ldots, D_{a^{+}}^{n-(n-\alpha)(1-\beta)-1, \beta} v_{k}\right), \\
& D_{a^{+}}^{\alpha, \beta} v_{k}(t)=\Psi\left(t, u_{k}, D_{a^{+}}^{1-(n-\alpha)(1-\beta), \beta} u_{k}, D_{a^{+}}^{2-(n-\alpha)(1-\beta), \beta} u_{k}, \ldots, D_{a^{+}}^{n-(n-\alpha)(1-\beta)-1, \beta} u_{k}\right) .
\end{aligned}
$$

Let us consider $u:=u_{1}-u_{2}$ and $v:=v_{1}-v_{2}$. It follows that

$$
\begin{aligned}
D_{a^{+}}^{\alpha, \beta} u(t)= & \Phi\left(t, v_{1}, D_{a^{+}}^{1-(n-\alpha)(1-\beta), \beta} v_{1}, D_{a^{+}}^{2-(n-\alpha)(1-\beta), \beta} v_{1}, \ldots, D_{a^{+}}^{n-(n-\alpha)(1-\beta)-1, \beta} v_{1}\right) \\
& -\Phi\left(t, v_{2}, D_{a^{+}}^{1-(n-\alpha)(1-\beta), \beta} v_{2}, D_{a^{+}}^{2-(n-\alpha)(1-\beta), \beta} v_{2}, \ldots, D_{a^{+}}^{n-(n-\alpha)(1-\beta)-1, \beta} v_{2}\right)
\end{aligned}
$$

and

$$
\begin{aligned}
D_{a^{+}}^{\alpha, \beta} v(t)= & \Psi\left(t, u_{1}, D_{a^{+}}^{1-(n-\alpha)(1-\beta), \beta} u_{1}, D_{a^{+}}^{2-(n-\alpha)(1-\beta), \beta} u_{1}, \ldots, D_{a^{+}}^{n-(n-\alpha)(1-\beta)-1, \beta} u_{1}\right) \\
& -\Psi\left(t, u_{2}, D_{a^{+}}^{1-(n-\alpha)(1-\beta), \beta} u_{2}, D_{a^{+}}^{2-(n-\alpha)(1-\beta), \beta} u_{2}, \ldots, D_{a^{+}}^{n-(n-\alpha)(1-\beta)-1, \beta} u_{2}\right) .
\end{aligned}
$$

Since $\operatorname{Im} \mathcal{L}=\operatorname{ker} Q$, we get that

$$
\begin{aligned}
& \int_{a}^{b}(b-s)^{-\beta(n-\alpha)} \\
& \quad \times\left\{\Phi\left(s, v_{1}, D_{a^{+}}^{1-(n-\alpha)(1-\beta), \beta} v_{1}, D_{a^{+}}^{2-(n-\alpha)(1-\beta), \beta} v_{1}, \ldots, D_{a^{+}}^{n-(n-\alpha)(1-\beta)-1, \beta} v_{1}\right)\right. \\
& \left.\quad-\Phi\left(s, v_{2}, D_{a^{+}}^{1-(n-\alpha)(1-\beta), \beta} v_{2}, D_{a^{+}}^{2-(n-\alpha)(1-\beta), \beta} v_{2}, \ldots, D_{a^{+}}^{n-(n-\alpha)(1-\beta)-1, \beta} v_{2}\right)\right\} d s=0
\end{aligned}
$$


and

$$
\begin{aligned}
& \int_{a}^{b}(b-s)^{-\beta(n-\alpha)} \\
& \quad \times\left\{\Psi\left(s, u_{1}, D_{a^{+}}^{1-(n-\alpha)(1-\beta), \beta} u_{1}, D_{a^{+}}^{2-(n-\alpha)(1-\beta), \beta} u_{1}, \ldots, D_{a^{+}}^{n-(n-\alpha)(1-\beta)-1, \beta} u_{1}\right)\right. \\
& \left.\quad-\Psi\left(s, u_{2}, D_{a^{+}}^{1-(n-\alpha)(1-\beta), \beta} u_{2}, D_{a^{+}}^{2-(n-\alpha)(1-\beta), \beta} u_{2}, \ldots, D_{a^{+}}^{n-(n-\alpha)(1-\beta)-1, \beta} u_{2}\right)\right\} d s=0 .
\end{aligned}
$$

Equalities (3.58) and (3.59) imply that there are $t_{2}, t_{3} \in[a, b]$ such that

$$
\begin{aligned}
& \Phi\left(t_{2}, v_{1}, D_{a^{+}}^{1-(n-\alpha)(1-\beta), \beta} v_{1}, D_{a^{+}}^{2-(n-\alpha)(1-\beta), \beta} v_{1}, \ldots, D_{a^{+}}^{n-(n-\alpha)(1-\beta)-1, \beta} v_{1}\right) \\
& \quad=\Phi\left(t_{2}, v_{2}, D_{a^{+}}^{1-(n-\alpha)(1-\beta), \beta} v_{2}, D_{a^{+}}^{2-(n-\alpha)(1-\beta), \beta} v_{2}, \ldots, D_{a^{+}}^{n-(n-\alpha)(1-\beta)-1, \beta} v_{2}\right)
\end{aligned}
$$

and

$$
\begin{aligned}
& \Psi\left(t_{3}, u_{1}, D_{a^{+}}^{1-(n-\alpha)(1-\beta), \beta} u_{1}, D_{a^{+}}^{2-(n-\alpha)(1-\beta), \beta} u_{1}, \ldots, D_{a^{+}}^{n-(n-\alpha)(1-\beta)-1, \beta} u_{1}\right) \\
& \quad=\Psi\left(t_{3}, u_{2}, D_{a^{+}}^{1-(n-\alpha)(1-\beta), \beta} u_{2}, D_{a^{+}}^{2-(n-\alpha)(1-\beta), \beta} u_{2}, \ldots, D_{a^{+}}^{n-(n-\alpha)(1-\beta)-1, \beta} u_{2}\right) .
\end{aligned}
$$

So, in view of hypothesis $\left(H_{2}^{\prime}\right)$, we get the following inequality:

$$
\begin{aligned}
0= & \mid \Phi\left(t_{2}, v_{1}, D_{a^{+}}^{1-(n-\alpha)(1-\beta), \beta} v_{1}, D_{a^{+}}^{2-(n-\alpha)(1-\beta), \beta} v_{1}, \ldots, D_{a^{+}}^{n-(n-\alpha)(1-\beta)-1, \beta} v_{1}\right) \\
& -\Phi\left(t_{2}, v_{2}, D_{a^{+}}^{1-(n-\alpha)(1-\beta), \beta} v_{2}, D_{a^{+}}^{2-(n-\alpha)(1-\beta), \beta} v_{2}, \ldots, D_{a^{+}}^{n-(n-\alpha)(1-\beta)-1, \beta} v_{2}\right) \mid \\
\geq & k_{n}\left|D_{a^{+}}^{n-(n-\alpha)(1-\beta)-1, \beta} v\left(t_{2}\right)\right|-k_{1}\left|v\left(t_{2}\right)\right|-\sum_{i=2}^{n-1} k_{i}\left|D_{a^{+}}^{n-(n-\alpha)(1-\beta)-i, \beta} v\left(t_{2}\right)\right| .
\end{aligned}
$$

Therefore this inequality gives us

$$
\left|D_{a^{+}}^{n-(n-\alpha)(1-\beta)-1, \beta} v\left(t_{2}\right)\right| \leq \frac{k_{1}}{k_{n}}\left|v\left(t_{2}\right)\right|+\sum_{i=2}^{n-1} \frac{k_{i}}{k_{n}}\left|D_{a^{+}}^{n-(n-\alpha)(1-\beta)-i, \beta} v\left(t_{2}\right)\right|,
$$

that is,

$$
\left|D_{a^{+}}^{n-(n-\alpha)(1-\beta)-1, \beta} v\left(t_{2}\right)\right| \leq \frac{k_{1}}{k_{n}}\left\|v\left(t_{2}\right)\right\|+\sum_{i=2}^{n-1} \frac{k_{i}}{k_{n}}\left\|D_{a^{+}}^{n-(n-\alpha)(1-\beta)-i, \beta} v\left(t_{2}\right)\right\| .
$$

From this inequality we immediately derive that

$$
\left|D_{a^{+}}^{n-(n-\alpha)(1-\beta)-1, \beta} v\left(t_{2}\right)\right| \leq \sum_{i=1}^{n-1} \frac{k_{i}}{k_{n}}\|v\|_{\mathcal{B}}
$$

Similarly, we can obtain

$$
\left|D_{a^{+}}^{n-(n-\alpha)(1-\beta)-1, \beta} u\left(t_{3}\right)\right| \leq \sum_{i=1}^{n-1} \frac{l_{i}}{l_{n}}\|u\|_{\mathcal{B}}
$$


Let us reconsider equalities (3.56) and (3.57). Accordingly, we have

$$
\begin{aligned}
D_{a^{+}}^{n-(n-\alpha)(1-\beta)-1, \beta} u(t) \\
=I_{a^{+}}^{1-\beta(n-\alpha)}\left\{\Phi\left(t_{2}, v_{1}, D_{a^{+}}^{1-(n-\alpha)(1-\beta), \beta} v_{1}, D_{a^{+}}^{2-(n-\alpha)(1-\beta), \beta} v_{1}, \ldots, D_{a^{+}}^{n-(n-\alpha)(1-\beta)-1, \beta} v_{1}\right)\right. \\
\left.\quad-\Phi\left(t_{2}, v_{2}, D_{a^{+}}^{1-(n-\alpha)(1-\beta), \beta} v_{2}, D_{a^{+}}^{2-(n-\alpha)(1-\beta), \beta} v_{2}, \ldots, D_{a^{+}}^{n-(n-\alpha)(1-\beta)-1, \beta} v_{2}\right)\right\} \\
\quad+D_{a^{+}}^{n-(n-\alpha)(1-\beta)-1, \beta} u(a)
\end{aligned}
$$

and

$$
\begin{aligned}
D_{a^{+}}^{n-(n-\alpha)(1-\beta)-1, \beta} v(t) \\
=I_{a^{+}}^{1-\beta(n-\alpha)}\left\{\Psi\left(t_{3}, u_{1}, D_{a^{+}}^{1-(n-\alpha)(1-\beta), \beta} u_{1}, D_{a^{+}}^{2-(n-\alpha)(1-\beta), \beta} u_{1}, \ldots, D_{a^{+}}^{n-(n-\alpha)(1-\beta)-1, \beta} u_{1}\right)\right. \\
\left.\quad-\Psi\left(t_{3}, u_{2}, D_{a^{+}}^{1-(n-\alpha)(1-\beta), \beta} u_{2}, D_{a^{+}}^{2-(n-\alpha)(1-\beta), \beta} u_{2}, \ldots, D_{a^{+}}^{n-(n-\alpha)(1-\beta)-1, \beta} u_{2}\right)\right\} \\
\quad+D_{a^{+}}^{n-(n-\alpha)(1-\beta)-1, \beta} v(a) .
\end{aligned}
$$

If we take $t=t_{3}$ in (3.64) and $t=t_{2}$ in (3.65), then (3.28), together with inequalities (3.62) and (3.63), yields

$$
\begin{aligned}
\left|D_{a^{+}}^{n-(n-\alpha)(1-\beta)-1, \beta} u(a)\right| & \leq \sum_{i=1}^{n-1} \frac{l_{i}}{l_{n}}\|u\|_{\mathcal{B}}+\Theta \sum_{i=1}^{n} \mu_{i}\|v\|_{\mathcal{B}}, \\
\left|D_{a^{+}}^{n-(n-\alpha)(1-\beta)-1, \beta} v(a)\right| & \leq \sum_{i=1}^{n-1} \frac{k_{i}}{k_{n}}\|v\|_{\mathcal{B}}+\Theta \sum_{i=1}^{n} v_{i}\|u\|_{\mathcal{B} .}
\end{aligned}
$$

Here we recall once again inequality (3.30):

$$
\begin{aligned}
\|(u, v)\|_{X}= & \|P(u, v)+(I-P)(u, v)\|_{X} \leq\|P(u, v)\|_{X}+\|(I-P)(u, v)\|_{X} \\
\leq & \max \left\{\left\{\Lambda_{1}\left|D_{a^{+}}^{n-(n-\alpha)(1-\beta)-1, \beta} u(a)\right|+\Lambda_{2}\left\|\mathcal{N}_{1} v\right\|_{\mathcal{B}}\right\},\right. \\
& \left\{\Lambda_{1}\left|D_{a^{+}}^{n-(n-\alpha)(1-\beta)-1, \beta} v(a)\right|+\Lambda_{2}\left\|\mathcal{N}_{2} u\right\|_{\mathcal{B}}\right\}, \\
& \left\{\Lambda_{1}\left|D_{a^{+}}^{n-(n-\alpha)(1-\beta)-1, \beta} u(a)\right|+\Lambda_{2}\left\|\mathcal{N}_{2} u\right\|_{\mathcal{B}}\right\}, \\
& \left.\left\{\Lambda_{1}\left|D_{a^{+}}^{n-(n-\alpha)(1-\beta)-1, \beta} v(a)\right|+\Lambda_{2}\left\|\mathcal{N}_{1} v\right\|_{\mathcal{B}}\right\}\right\} .
\end{aligned}
$$

Similarly to case i, (3.32), case ii, (3.34), case iii, (3.36), and case iv, (3.38), we conclude that

$$
\begin{aligned}
\|(u, v)\|_{X} & \leq \frac{\Lambda_{2} d_{1}}{1-\left[\Lambda_{1}\left\{\sum_{i=1}^{n-1} \frac{l_{i}}{l_{n}}+\Theta \sum_{i=1}^{n} \mu_{i}\right\}+\Lambda_{2} \sum_{i=1}^{n} b_{i}\right]}, \\
\|(u, v)\|_{X} & \leq \frac{\Lambda_{2} d_{2}}{1-\left[\Lambda_{1}\left\{\sum_{i=1}^{n-1} \frac{k_{i}}{k_{n}}+\Theta \sum_{i=1}^{n} v_{i}\right\}+\Lambda_{2} \sum_{i=1}^{n} c_{i}\right]}, \\
\|(u, v)\|_{X} & \leq \frac{\Lambda_{2} d_{2}}{1-\left[\Lambda_{1}\left\{\sum_{i=1}^{n-1} \frac{l_{i}}{l_{n}}+\Theta \sum_{i=1}^{n} \mu_{i}\right\}+\Lambda_{2} \sum_{i=1}^{n} c_{i}\right]},
\end{aligned}
$$

and

$$
\|(u, v)\|_{X} \leq \frac{\Lambda_{2} d_{1}}{1-\left[\Lambda_{1}\left\{\sum_{i=1}^{n-1} \frac{k_{i}}{k_{n}}+\Theta \sum_{i=1}^{n} v_{i}\right\}+\Lambda_{2} \sum_{i=1}^{n} b_{i}\right]} .
$$


Finally, if we impose conditions (3.51)-(3.54) into inequalities (3.68)-(3.71), respectively, then it follows that $u=v=0$. More precisely, we have proven that $u_{1}=u_{2}$ and $v_{1}=v_{2}$. This completes the proof of uniqueness of the solutions of the Hilfer fractional resonant system (1.1)-(1.2).

\section{An application}

In this section, we present an application to illustrate the obtained theoretical existence and uniqueness criteria in the frame of Theorems 3.8 and 3.9.

Example 4.1 Let us consider the Hilfer fractional resonant system

$$
\begin{cases}D_{0^{+}}^{\frac{5}{2}, \frac{1}{2}} u(t)=\Phi\left(t, v, D_{0^{+}}^{\frac{1}{2}, \frac{1}{2}} v, D_{0^{+}}^{\frac{3}{2}, \frac{1}{2}} v\right), & 0<t<1, \\ D_{0^{+}}^{\frac{5}{2}, \frac{1}{2}} v(t)=\Psi\left(t, u, D_{0^{+}}^{\frac{1}{2}, \frac{1}{2}} u, D_{0^{+}}^{\frac{3}{2}, \frac{1}{2}} u\right), & 0<t<1,\end{cases}
$$

with nonlinearities

$$
\begin{aligned}
& \Phi\left(t, x_{1}, x_{2}, x_{3}\right):=\frac{t+\left|x_{1}\right|+\left|x_{2}\right|+\left|x_{3}\right|}{10}, \\
& \Psi\left(t, x_{1}, x_{2}, x_{3}\right):=\frac{t+\left|\sin \left(x_{1}\right)\right|+\left|\sin \left(x_{2}\right)\right|+\left|\sin \left(x_{3}\right)\right|}{10},
\end{aligned}
$$

subject to the boundary conditions

$$
\left\{\begin{array}{lll}
I_{0^{+}}^{0.25,0.5} u(0)=0, & D_{0^{+}}^{0.75,0.5} u(0)=0, & D_{0^{+}}^{1.75,0.5} u(0)=D_{0^{+}}^{1.75,0.5} u(1), \\
I_{0^{+}}^{0.25,0.5} v(0)=0, & D_{0^{+}}^{0.75,0.5} v(0)=0, & D_{0^{+}}^{1.75,0.5} v(0)=D_{0^{+}}^{1.75,0.5} v(1) .
\end{array}\right.
$$

Indeed, in the Hilfer fractional resonant system (1.1)-(1.2), we impose the following setting to reach the fractional resonant system (4.1)-(4.3):

$$
\alpha:=2.5, \quad \beta:=0.5, \quad n=3, \quad a:=0, \quad b:=1 .
$$

By a direct calculation we get that

$$
\Lambda_{1}:=1, \quad \Lambda_{2}=\Theta:=0.100946
$$

Finally, taking $B:=1, \eta_{i}=\zeta_{i}:=1, i=1,2,3$, and $k_{i}=l_{i}:=0.1, i=1,2,3$, it is easy to check that the hypotheses $\left(H_{2}\right)-\left(H_{3}\right)$ and $\left(H_{1}^{\prime}\right)-\left(H_{3}^{\prime}\right)$ are satisfied. So, the Hilfer fractional resonant system (4.1)-(4.3) has a unique solution on $X$.

\section{Discussion and concluding remarks}

In this paper, we studied the higher-order Hilfer fractional resonant system (1.1)-(1.2). Our aim in this investigation was to apply the coincidence degree theory to obtain at least one solution for the resonant system (1.1)-(1.2). Besides, having certain conditions on the nonlinearities, we presented a uniqueness criterion. One of the advantages of this investigation is that, to the best of our knowledge, this is the first time in the literature that the Hilfer fractional differential equations have been considered to establish the fractional resonant problems. 
At this position, we discuss other advantages of the Hilfer fractional derivatives. As mentioned in Definition 2.4, taking $\beta=0$ gives us the Riemann-Liouville fractional resonant system

$$
\left\{\begin{array}{lr}
D_{a^{+}}^{\alpha} u(t)=\Phi\left(t, v, D_{a^{+}}^{\alpha-n+1} v, D_{a^{+}}^{\alpha-n+2} v, \ldots, D_{a^{+}}^{\alpha-1} v\right), & a<t<b, \\
D_{a^{+}}^{\alpha} v(t)=\Psi\left(t, u, D_{a^{+}}^{\alpha-n+1} u, D_{a^{+}}^{\alpha-n+2} u, \ldots, D_{a^{+}}^{\alpha-1} u\right), & a<t<b,
\end{array}\right.
$$

subject to the boundary conditions

$$
\left\{\begin{array}{l}
I_{a^{+}}^{n-\alpha} u(a)=0, \quad D_{a^{+}}^{\alpha-i} u(a)=0, \\
D_{a^{+}}^{\alpha-1} u(a)=D_{a^{+}}^{\alpha-1} u(b), \quad i=2,3, \ldots, n-1, \\
I_{a^{+}}^{n-\alpha} v(a)=0, \quad D_{a^{+}}^{\alpha-i} v(a)=0, \\
D_{a^{+}}^{\alpha-1} v(a)=D_{a^{+}}^{\alpha-1} v(b), \quad i=2,3, \ldots, n-1,
\end{array}\right.
$$

which generalizes all the Riemann-Liouville-based fractional resonant problems with boundary conditions of the form (5.2).

Also, taking $\beta:=1$, we get the following Caputo fractional resonant system:

$$
\begin{cases}{ }^{c} D_{a^{+}}^{\alpha} u(t)=\Phi\left(t, v, v^{\prime}, v^{\prime \prime}, \ldots, v^{(n-1)}\right), & a<t<b, \\ { }^{c} D_{a^{+}}^{\alpha} v(t)=\Psi\left(t, u, u^{\prime}, u^{\prime \prime}, \ldots, u^{(n-1)}\right), & a<t<b,\end{cases}
$$

subject to the boundary conditions

$$
\left\{\begin{array}{l}
u(a)=u^{\prime}(a)=u^{\prime \prime}(a)=\cdots=u^{(n-2)}(a)=0, \quad u^{(n-1)}(a)=u^{(n-1)}(b), \\
v(a)=v^{\prime}(a)=v^{\prime \prime}(a)=\cdots=v^{(n-2)}(a)=0,
\end{array}\right.
$$

which coincides with the main problem in [19], that is, (1.7)-(1.8), and it generalizes [20] and [39].

We believe that due to the unifying characteristics of the Hilfer fractional derivatives, differential equations equipped with this generalized derivatives have much more potential to reach new results both in the theory and in the applications of the fractional-order problems. For instance, as a future research works, one may consider the half-linear Hilfer fractional differential equations and try to extract the corresponding Lyapunov-type inequalities to describe the qualitative dynamics of these problems involving stability, disconjugacy, nonexistence, algebraic properties of nontrivial solutions, and so on. Furthermore, applicability of the half-linear dynamical systems in the porous medium opens a new research line to find more applications of the Hilfer fractional dynamical systems.

\section{Acknowledgements}

The author would like to appreciate the editorial board and both of the anonymous referees for their detailed and invaluable suggestions on the first draft.

Funding

Not applicable. 


\section{Competing interests}

The author declares that he has no competing interests regarding the publication of this paper.

\section{Authors' contributions}

The author read and approved the final version of the current paper.

\section{Publisher's Note}

Springer Nature remains neutral with regard to jurisdictional claims in published maps and institutional affiliations.

Received: 22 July 2020 Accepted: 31 August 2020 Published online: 09 September 2020

\section{References}

1. Abbas, S., Agarwal, R.P., Benchohra, M., Lazreg, J., Ahmad, B.: Bounded weak solutions for Hilfer fractional differential equations on the half line. Adv. Dyn. Syst. Appl. 15(1), 35-49 (2020)

2. Abbas, S., Benchohra, M., Lazreg, J., Zhou, Y.: A survey on Hadamard and Hilfer fractional differential equations: analysis and stability. Chaos Solitons Fractals 102, 47-71 (2017)

3. Agrawal, R.P., O'Regan, D., Staněk, S.: Positive solutions for Dirichlet problem of singular nonlinear fractional differential equations. J. Math. Anal. Appl. 371, 57-68 (2010)

4. Atanackovic, T.M., Pilipovic, S., Stankovic, B., Zorica, D.: Fractional Calculus with Applications in Mechanics. Wiley, New York (2014)

5. Bai, Z., Lu, H.: Positive solutions for boundary value problem of nonlinear fractional differential equations. J. Math. Anal. Appl. 311, 495-505 (2005)

6. Baleanu, D., Guvenc, Z.B., Machado, J.A.T.: New Trends in Nanotechnology and Fractional Calculus Applications. Springer, Berlin (2010)

7. Baleanu, D., Machado, J.A.T., Luo, A.C.J.: Fractional Dynamics and Control. Springer, Berlin (2012)

8. Bhairat, S.P.: Existence and continuation of solutions of Hilfer fractional differential equations. J. Math. Model. 7(1), $1-20(2019)$

9. Bhalekar, S., Daftardar-Gejji, V.: New iterative method: application to partial differential equations. Appl. Math. Comput. 203, 778-783 (2008)

10. Choudhary, S., Daftardar-Gejji, V.: Nonlinear multi-order fractional differential equations with periodic/antiperiodic boundary conditions. Fract. Calc. Appl. Anal. 17(2), 333-347 (2014). https://doi.org/10.2478/s13540-014-0172-6

11. Daftardar-Gejji, V., Bhalekar, S.: Solving fractional boundary value problems with Dirichlet boundary conditions using a new iterative method. Comput. Math. Appl. 59, 1801-1809 (2010)

12. Darwish, M.A.: On Erdelyi-Kober fractional Urysohn-Volterra quadratic integral equations. Appl. Math. Comput. 273, 562-569 (2016)

13. Dhaigude, C.D., Nikam, V.R.: Solution of fractional partial differential equations using iterative method. Fract. Calc. Appl. Anal. 15(4), 684-699 (2012). https://doi.org/10.2478/s13540-012-0046-8

14. Duong, P.L.T., Kwok, E., Lee, M.: Deterministic analysis of distributed order systems using operational matrix. Appl. Math. Model. 40(3), 1929-1940 (2016)

15. Gaines, R.E., Mawhin, J.: Coincidence Degree and Nonlinear Differential Equations. Springer, Berlin (1977)

16. Gholami, Y., Ghanbari, K.: Coupled systems of Caputo type fractional $\Delta$-difference boundary value problems at resonance. Trans. A. Razmadze Math. Inst. 171, 332-349 (2017)

17. Gu, H., Trujillo, J.J.: Existence of mild solution for evolution equation with Hilfer fractional derivative. Appl. Math. Comput. 257, 344-354 (2015)

18. Hilfer, R.: Applications of Fractional Calculus in Physics. World Scientific, Singapore (2000)

19. Hu, L., Zhang, S.: Existence and uniqueness of solutions for a higher-order coupled fractional differential equations at resonance. Adv. Differ. Equ. 2015, 202 (2015)

20. Hu, Z., Liu, W., Chen, T.: Existence of solutions for a coupled system of fractional differential equations at resonance. Bound. Value Probl. 2012, 98 (2012)

21. Jaiswal, A., Bahuguna, D.: Hilfer fractional differential equations with almost sectorial operators. Differ. Equ. Dyn. Syst. (2020). https://doi.org/10.1007/s12591-020-00514-y

22. Jiang, W.: The existence of solutions to boundary value problems of fractional differential equations at resonance. Nonlinear Anal. 74, 1987-1994 (2011)

23. Kamocki, R.: A new representation formula for the Hilfer fractional derivative and its application. J. Comput. Appl. Math. 308, 39-45 (2016)

24. Kavitha, K., Vijayakumar, V., Udhayakumar, R.: Results on controllability of Hilfer fractional neutral differential equations with infinite delay via measures of noncompactness. Chaos Solitons Fractals 139, 110035 (2020)

25. Kexue, L., Jigen, P., Jinghuai, G.: Existence results for semilinear fractional differential via Kuratowski measure of noncompactness. Fract. Calc. Appl. Anal. 15(4), 591-610 (2012). https://doi.org/10.2478/s13540-012-0041-0

26. Kilbas, A.A., Srivastava, H.M., Trujillo, J.J.: Theory and Applications of Fractional Differential Equations. North-Holland Mathematics Studies, vol. 204. Elsevier, Amsterdam (2006)

27. Kiryakova, V.: Generalized Fractional Calculus and Applications. Pitman, London (1994)

28. Kosmatov, N.: Multi-point boundary value problems on time scales at resonance. J. Math. Anal. Appl. 323, 253-266 (2006)

29. Kosmatov, N.: A boundary value problem of fractional order at resonance. Electron. J. Differ. Equ. 2010, 135 (2010)

30. Kosmatov, N.: A singular non-local problem at resonance. J. Math. Anal. Appl. 394, 425-431 (2012)

31. Kosmatov, N., Jiang, W.: Resonant functional problems of fractional order. Chaos Solitons Fractals 91, 573-579 (2016)

32. LV, J., Yang, X.: Approximate controllability of Hilfer fractional differential equations. Math. Methods Appl. Sci. 43(1), 242-254 (2020)

33. Mainardi, F:: Fractional Calculus and Waves in Linear Viscoelasticity. World Scientific, Singapore (2010)

34. Miller, K.S., Ross, B.: An Introduction to Fractional Calculus and Fractional Differential Equation. Wiley, New York (1993) 
35. Oldham, K.B., Spanier, J.: The Fractional Calculus. Academic Press, New York (1974)

36. Petras, I.: Fractional-Order Nonlinear Systems. Modeling, Analysis and Simulation. Springer, Berlin (2011)

37. Podlubny, I.: Fractional Differential Equations. Mathematics in Science and Applications, vol. 19. Academic Press, New York (1999)

38. Ray, S.S.: Fractional Calculus with Applications for Nuclear Reactor Dynamics. CRC Press, Boca Raton (2016)

39. Rui, W.: Existence of solutions of nonlinear fractional differential equations at resonance. Electron. J. Qual. Theory Differ. Equ. 2011, 66 (2011)

40. Subashini, R., Jothimani, K., Sooppy Nisar, K., Ravichandran, C.: New results on nonlocal functional integro-differential equations via Hilfer fractional derivative. Alex. Eng. J. (2020). https://doi.org/10.1016/j.aej.2020.01.055

41. Tomovski, Z:: Generalized Cauchy type problems for nonlinear fractional differential equations with composite fractional derivative operator. Nonlinear Anal. TMA 75, 3364-3384 (2012)

42. ur Rehman, M., Saeed, U.: Gegenbauer wavelet operational matrix method for fractional differential equations. J. Korean Math. Soc. 52(5), 1069-1096 (2015)

43. Vijayakumar, V., Udhayakumar, R.: Results on approximate controllability for non-densely defined Hilfer fractional differential system with infinite delay. Chaos Solitons Fractals 139, 110019 (2020)

44. Wang, J.R., Zhang, Y.: Nonlocal initial value problems for differential equations with Hilfer fractional derivative. Appl. Math. Comput. 266, 850-859 (2015)

45. Yi, M., Wang, L., Huang, J.: Legendre wavelets method for the numerical solution of fractional integro-differential equations with weakly singular kernel. Appl. Math. Model. 40(4), 3422-3437 (2016)

46. Zhang, S.: Existence of positive solutions for some class of fractional differential equations. J. Math. Anal. Appl. 278, $136-148$ (2003)

47. Zhang, S., Han, G.: The existence of a positive solution for a nonlinear fractional differential equation. J. Math. Anal. Appl. 252, 804-812 (2000)

48. Zhang, Y., Bai, Z.: Existence of solutions for nonlinear fractional three-point boundary value problems at resonance. J. Appl. Math. Comput. 36, 417-440 (2011)

\section{Submit your manuscript to a SpringerOpen ${ }^{\circ}$ journal and benefit from:}

- Convenient online submission

- Rigorous peer review

- Open access: articles freely available online

- High visibility within the field

- Retaining the copyright to your article

Submit your next manuscript at $\gg$ springeropen.com 